JOURNAL OF

SYMPLECTIC GEOMETRY

Volume 11, Number 1, 1-24, 2013

\title{
ON REGULAR COURANT ALGEBROIDS
}

\author{
Zhuo Chen, Mathieu Stiénon, and Ping Xu
}

For any regular Courant algebroid, we construct a characteristic class à la Chern-Weil. This intrinsic invariant of the Courant algebroid is a degree- 3 class in its naive cohomology. When the Courant algebroid is exact, it reduces to the Ševera class in $H_{\mathrm{DR}}^{3}(M)$. On the other hand, when the Courant algebroid is a quadratic Lie algebra $\mathfrak{g}$, it coincides with the class of the Cartan 3-form in $H^{3}(\mathfrak{g})$. We also give a complete classification of regular Courant algebroids and discuss its relation to the characteristic class.

\section{Introduction}

Courant algebroids were introduced in $[\mathbf{1 0}]$ as a way to merge the concept of Lie bialgebra and the bracket on $\mathfrak{X}(M) \oplus \Omega^{1}(M)$ first discovered by Courant $[\mathbf{3}]$ - here $M$ is a smooth manifold. Roytenberg gave an equivalent definition phrased in terms of the Dorfman bracket [12], which highlighted the relation of Courant algebroids to $L_{\infty}$-algebras [16] observed by Roytenberg and Weinstein [14].

Despite its importance, the subject suffered from the lack of examples for a long time. One important class of Courant algebroids, called exact Courant algebroids, was discovered by Ševera [15]. A Courant algebroid $E$ is said to be exact if its underlying vector bundle fits into an exact sequence $0 \rightarrow T^{*} M \stackrel{\rho^{*}}{\longrightarrow} E \stackrel{\rho}{\rightarrow} T M \rightarrow 0$, where $\rho$ is the anchor map. Ševera proved that the isomorphism classes of exact Courant algebroids are classified by a degree-3 de Rham cohomology class of $M$ : the Ševera class. Furthermore, the structure of transitive Courant algebroids - Courant algebroids with surjective anchors - was described independently by Vaisman [18], Ševera (in a private correspondence with Weinstein [15]) and Bressler [2]. Ševera and Bressler also classified transitive Courant algebroids as extensions of transitive Lie algebroids $[\mathbf{2 , 1 5}$. Indeed Ševera also outlined some nice ideas of classification of transitive Courant algebroids in [15]. 
In [17], the second and third authors introduced the modular class of Courant algebroids, a degree- 1 characteristic class in the naive cohomology $H_{\text {naive }}^{1}(E)$ of the Courant algebroid $E$. It is natural to ask whether there is a degree-3 characteristic class for a general Courant algebroid resembling the Ševera class, and if so, what role is played by such a class in the classification of Courant algebroids. The main purpose of this paper is to answer these questions for regular Courant algebroids, that is, Courant algebroids with a constant rank anchor. Such Courant algebroids $E$ are particularly easy to handle, for their associated characteristic distribution $\rho(E)$ does not have any singularity. Note that characteristic classes of Lie algebroids were studied by Evens et al. [5], Crainic and Fernandes [4,6], and Gracia-Saz and Mehta $[8]$. It would be interesting to explore if there is any intrinsic connection between these constructions.

For any regular Courant algebroid, we will construct a degree-3 class, called the characteristic class, in the naive cohomology $H_{\text {naive }}^{3}(E)$ of the Courant algebroid, an analogue of Ševera's class. Note that when $E$ is an exact Courant algebroid, $H_{\text {naive }}^{3}(E)$ is isomorphic to the de Rham cohomology $H_{\mathrm{DR}}^{3}(M)$. However, unlike the exact case, $H_{\text {naive }}^{3}(E)$ does not classify regular Courant algebroids. The classification problem is much subtler in this case.

Given a regular Courant algebroid $E$ with anchor $\rho$, the quotient bundle $\mathcal{A}:=E /(\operatorname{ker} \rho)^{\perp}$ is clearly a regular Lie algebroid, i.e., a Lie algebroid with a constant rank anchor. It is called the ample Lie algebroid of $E$. The kernel, $\mathcal{G}=\operatorname{ker} a$ of the anchor $a$ of $\mathcal{A}$, is a bundle of quadratic Lie algebras, which satisfies certain compatibility conditions. Therefore, the Lie algebroid $\mathcal{A}$ is quadratic (see Definition 1.8).

The first natural question is whether every quadratic Lie algebroid $\mathcal{A}$ arises in this way. It turns out that there is an obstruction: the first Pontryagin class, an element in $H^{4}(F)$ naturally associated to any quadratic Lie algebroid. Here $F$ is the image of the anchor (an integrable subbundle of $T M)$ and $H^{4}(F)$ stands for the leafwise de Rham cohomology of $F$. This obstruction is similar to the one described by Ševera and Bressler in the transitive case $[\mathbf{2}, \mathbf{1 5}]$.

To recover a Courant algebroid from a given quadratic Lie algebroid $\mathcal{A}$ with vanishing first Pontryagin class, one needs an extra piece of data: a coherent form $C$. This is a closed 3 -form on the Lie algebroid $\mathcal{A}$ satisfying certain compatibility conditions. In this case $(\mathcal{A}, C)$ is called a characteristic pair.

An equivalence is introduced on characteristic pairs. Let $H_{\leftrightarrow}^{\bullet}(\mathcal{A})$ denote the cohomology groups of the subcomplex $\mathbf{C}_{\leftrightarrow}^{\bullet}(\mathcal{A})$ of $\left(\Gamma\left(\wedge^{\bullet} \mathcal{A}^{*}\right), d\right)$, where $\mathbf{C}_{\leftrightarrow}^{k}(\mathcal{A})$ consists of the sections of $\wedge^{k} \mathcal{A}^{*}$ which are annihilated by all sections of $\wedge^{k} \mathcal{G}$. Roughly speaking, two coherent forms $C_{1}$ and $C_{2}$ on $\mathcal{A}$ are equivalent if and only if the class of $C_{1}-C_{2}$ in $H_{\leftrightarrow}^{3}(\mathcal{A})$ is zero. We prove that there 
is a one-to-one correspondence between regular Courant algebroids up to isomorphism and equivalence classes of characteristic pairs.

The inclusion $i: \mathcal{G} \rightarrow \mathcal{A}$ is a Lie algebroid morphism. Therefore, it induces a morphism $i^{*}: H^{3}(\mathcal{A}) \rightarrow H^{3}(\mathcal{G})$. We denote the class of the standard Cartan 3-form of Lie theory in $H^{3}(\mathcal{G})$ by $\alpha$.

The main result of this paper can be summarized as the following:

Theorem. (1) There is a natural map from regular Courant algebroids to quadratic Lie algebroids with vanishing first Pontryagin class.

(2) For any quadratic Lie algebroid $\mathcal{A}$ with vanishing first Pontryagin class, the isomorphism classes of Courant algebroids whose ample Lie algebroids are isomorphic to $\mathcal{A}$ are parameterized by $\frac{a^{*} H^{3}(F)}{\mathbb{I}} \cong$ $\frac{H^{3}(F)}{\left(a^{*}\right)^{-1}(\mathbb{I})}$. Here $\mathbb{I}$ is a certain abelian subgroup of $a^{*} H^{3}(F) \subset H_{\leftrightarrow}^{3}(\mathcal{A})$ and a denotes the anchor of $\mathcal{A}$.

(3) For any regular Courant algebroid $E$, there is a degree-3 characteristic class, which is a cohomology class in $\left(i^{*}\right)^{-1}(\alpha) \subset H^{3}(\mathcal{A})$. Here $\mathcal{A}$ is the ample Lie algebroid of $E$, and $\alpha \in H^{3}(\mathcal{G})$ is the Cartan 3-form.

Hence, we have the "exact sequence"

isomorphism classes of
regular Courant algebroids
with characteristic distri-
bution $F$

$\stackrel{\Phi}{\rightarrow} \begin{aligned} & \text { isomorphism classes of } \\ & \text { quadratic Lie algebroids } \\ & \text { with characteristic distri- } \\ & \text { bution } F\end{aligned} \stackrel{\mathrm{FPC}}{\longrightarrow} H^{4}(F)$

Here "FPC" stands for the first Pontryagin class as defined in Lemma 1.9.

To this day, little is known about Courant algebroid cohomology [13]: Roytenberg computed it for $T M \oplus T^{*} M$ and Ginot and Grützmann for transitive and some very special regular Courant algebroids [7]. Our result should be useful for computing the Courant algebroid cohomology of arbitrary regular Courant algebroids. We remark that the relation between our work and the so called matched pairs of Courant algebroids are recently investigated by Grützmann and one of the authors $[\mathbf{9}]$.

The reader is assumed to be familiar with the Lie algebroid theory $[\mathbf{1 1}$.

\section{Preliminaries}

1.1. Regular Courant algebroids. A Courant algebroid consists of a vector bundle $E \rightarrow M$, a fiberwise nondegenerate pseudo-metric $(-,-)$, a bundle map $\rho: E \rightarrow T M$ called anchor and an $\mathbb{R}$-bilinear operation $\circ$ on $\Gamma(E)$ called the Dorfman bracket, which, for all $f \in C^{\infty}(M)$ and $e_{1}, e_{2}, e_{3} \in \Gamma(E)$ 
satisfy the following relations:

$$
\begin{gathered}
e_{1} \circ\left(e_{2} \circ e_{3}\right)=\left(e_{1} \circ e_{2}\right) \circ e_{3}+e_{2} \circ\left(e_{1} \circ e_{3}\right), \\
\rho\left(e_{1} \circ e_{2}\right)=\left[\rho\left(e_{1}\right), \rho\left(e_{2}\right)\right], \\
e_{1} \circ\left(f e_{2}\right)=\left(\rho\left(e_{1}\right) f\right) e_{2}+f\left(e_{1} \circ e_{2}\right), \\
\frac{1}{2}\left(e_{1} \circ e_{2}+e_{2} \circ e_{1}\right)=\mathcal{D}\left(e_{1}, e_{2}\right), \\
\mathcal{D} f \circ e_{1}=0, \\
\rho\left(e_{1}\right)\left(e_{2}, e_{3}\right)=\left(e_{1} \circ e_{2}, e_{3}\right)+\left(e_{2}, e_{1} \circ e_{3}\right),
\end{gathered}
$$

where $\mathcal{D}: C^{\infty}(M) \rightarrow \Gamma(E)$ is the $\mathbb{R}$-linear map defined by

$$
(\mathcal{D} f, e)=\frac{1}{2} \rho(e) f .
$$

The symmetric part of the Dorfman bracket is given by equation (1.4). The Courant bracket is defined as the skew-symmetric part

$$
\llbracket e_{1}, e_{2} \rrbracket=\frac{1}{2}\left(e_{1} \circ e_{2}-e_{2} \circ e_{1}\right)
$$

of the Dorfman bracket. Thus, we have the relation $e_{1} \circ e_{2}=\llbracket e_{1}, e_{2} \rrbracket+$ $\mathcal{D}\left(e_{1}, e_{2}\right)$.

Using the identification $\Xi: E \rightarrow E^{*}$ induced by the pseudo-metric $(-,-)$ :

$$
\left\langle\Xi\left(e_{1}\right) \mid e_{2}\right\rangle:=\left(e_{1}, e_{2}\right), \quad \forall e_{1}, e_{2} \in E,
$$

we can rewrite equation (1.7) as

$$
\mathcal{D} f=\frac{1}{2} \Xi^{-1} \rho^{*} d f \text {. }
$$

It is easy to see that $(\operatorname{ker} \rho)^{\perp}$, the subbundle of $E$ orthogonal to ker $\rho$ with respect to the pseudo-metric, coincides with $\Xi^{-1} \rho^{*}\left(T^{*} M\right)$, the subbundle of $E$ generated by the image of $\mathcal{D}: C^{\infty}(M) \rightarrow \Gamma(E)$. From equation (1.6), it follows that $\rho(\mathcal{D} f)=0$ for any $f \in C^{\infty}(M)$. Therefore, the kernel of the anchor is coisotropic:

$$
(\operatorname{ker} \rho)^{\perp} \subset \operatorname{ker} \rho \text {. }
$$

The spaces of sections of $\operatorname{ker} \rho$ and $(\operatorname{ker} \rho)^{\perp}$ are two-sided ideals of $\Gamma(E)$ with respect to the Dorfman bracket.

A Courant algebroid $E$ is said to be regular if $F:=\rho(E)$ has constant rank, in which case $F$ is an integrable distribution on the base manifold $M$. Moreover, if $E$ is regular, then $\operatorname{ker} \rho$ and $(\operatorname{ker} \rho)^{\perp}$ are smooth (constant rank) subbundles of $E$ and the quotients $E / \operatorname{ker} \rho$ and $E /(\operatorname{ker} \rho)^{\perp}$ are Lie algebroids. Obviously, $E / \operatorname{ker} \rho$ and $F$ are canonically isomorphic. We call $E /(\operatorname{ker} \rho)^{\perp}$ the ample Lie algebroid associated to $E$. It will be denoted by the symbol $\mathcal{A}_{E}$.

The inclusions

$$
(\operatorname{ker} \rho)^{\perp} \subset \operatorname{ker} \rho \subset E
$$


yield four exact sequences:

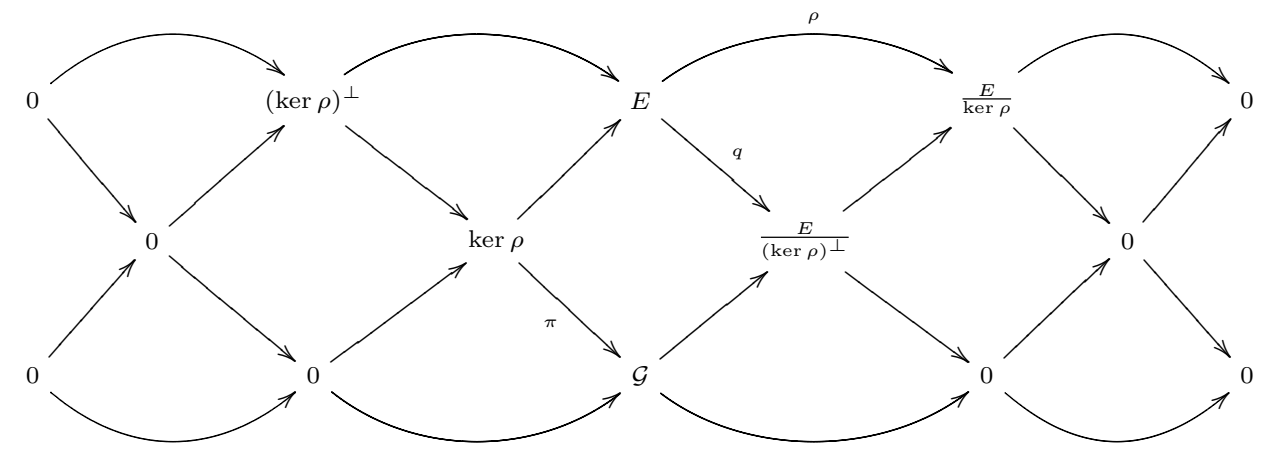

Here, $\mathcal{G}:=\operatorname{ker} \rho /(\operatorname{ker} \rho)^{\perp}$. We use the symbol $\pi$ (resp. $q$ ) to denote the projection $\operatorname{ker} \rho \rightarrow \mathcal{G}\left(\right.$ resp. $\left.E \rightarrow \mathcal{A}_{E}=E /(\operatorname{ker} \rho)^{\perp}\right)$.

1.2. Bundle of quadratic Lie algebras. A $C^{\infty}(M)$-bilinear and skewsymmetric bracket on $\Gamma(\mathcal{G})$ determined by the Dorfman bracket of $E$ through the relation

$$
[\pi(r), \pi(s)]^{\mathcal{G}}=\pi(r \circ s), \quad \forall r, s \in \Gamma(\operatorname{ker} \rho),
$$

turns $\mathcal{G}$ into a bundle of Lie algebras. Moreover, the map

$$
\pi(r) \otimes \pi(s) \mapsto(r, s)
$$

is a well-defined nondegenerate symmetric and ad-invariant pseudo-metric on $\mathcal{G}$, which we will denote by the symbol $(-,-)^{\mathcal{G}}$. Hence, $\mathcal{G}$ is a bundle of quadratic Lie algebras ${ }^{1}$.

Note that $\mathcal{G}$ is also a module over $\mathcal{A}_{E}$; the representation is given by

$$
x \cdot \pi(r)=[x, q(r)], \quad \forall x \in \Gamma\left(\mathcal{A}_{E}\right), r \in \Gamma(\operatorname{ker} \rho) .
$$

This representation is compatible with the pseudo-metric on $\mathcal{G}$ :

$$
a(x)(\boldsymbol{r}, \boldsymbol{s})^{\mathcal{G}}=(x \cdot \boldsymbol{r}, \boldsymbol{s})^{\mathcal{G}}+(\boldsymbol{r}, x \cdot \boldsymbol{s})^{\mathcal{G}}, \quad \forall x \in \Gamma\left(\mathcal{A}_{E}\right), \boldsymbol{r}, \boldsymbol{s} \in \Gamma(\mathcal{G}) .
$$

1.3. Dissections of regular Courant algebroids. Let $E$ be a regular Courant algebroid with characteristic distribution $F$ and bundle of quadratic Lie algebras $\mathcal{G}$. A dissection of $E$ is an isomorphism of vector bundles

$$
\Psi: F^{*} \oplus \mathcal{G} \oplus F \rightarrow E
$$

such that

$$
(\Psi(\xi+\boldsymbol{r}+x), \Psi(\eta+\boldsymbol{s}+y))=\frac{1}{2}\langle\xi \mid y\rangle+\frac{1}{2}\langle\eta \mid x\rangle+(\boldsymbol{r}, \boldsymbol{s})^{\mathcal{G}},
$$

for all $\xi, \eta \in \Gamma\left(F^{*}\right), \boldsymbol{r}, \boldsymbol{s} \in \Gamma(\mathcal{G})$ and $x, y \in \Gamma(F)$. Such an isomorphism transports the Courant algebroid structure of $E$ to $F^{*} \oplus \mathcal{G} \oplus F$.

\footnotetext{
${ }^{1} \mathrm{~A}$ Lie algebra is said to be quadratic if there exists a nondegenerate, ad-invariant pseudo-metric on its underlying vector space.
} 
Each dissection of $E$ induces three canonical maps:

(1) $\nabla: \Gamma(F) \otimes \Gamma(\mathcal{G}) \rightarrow \Gamma(\mathcal{G}):$

$$
\nabla_{x} \boldsymbol{r}=\operatorname{Pr}_{\mathcal{G}}(x \circ \boldsymbol{r}), \quad \forall x \in \Gamma(F), \boldsymbol{r} \in \Gamma(\mathcal{G}) ;
$$

(2) $R: \Gamma(F) \otimes \Gamma(F) \rightarrow \Gamma(\mathcal{G}):$

$$
R(x, y)=\operatorname{Pr}_{\mathcal{G}}(x \circ y), \quad \forall x, y \in \Gamma(F) ;
$$

(3) $\mathcal{H}: \Gamma(F) \otimes \Gamma(F) \otimes \Gamma(F) \rightarrow C^{\infty}(M):$

$$
\mathcal{H}(x, y, z)=\left\langle\operatorname{Pr}_{F^{*}}(x \circ y) \mid z\right\rangle, \quad \forall x, y, z \in \Gamma(F) .
$$

Proposition 1.1. (1) The map $\nabla$ satisfies

$$
\nabla_{f x} \boldsymbol{r}=f \nabla_{x} \boldsymbol{r} \quad \text { and } \quad \nabla_{x}(f \boldsymbol{r})=f \nabla_{x} \boldsymbol{r}+(x(f)) \boldsymbol{r},
$$

for all $x \in \Gamma(F), \boldsymbol{r} \in \Gamma(\mathcal{G})$ and $f \in C^{\infty}(M)$.

(2) The map $R$ is skew-symmetric and $C^{\infty}(M)$-bilinear. It can thus be regarded as a bundle map $\wedge^{2} F \rightarrow \mathcal{G}$.

(3) The map $\mathcal{H}$ is skew-symmetric and $C^{\infty}(M)$-bilinear. It can thus be regarded as a section of $\wedge^{3} F^{*}$.

The following lemma shows that dissections always exist.

Lemma 1.2. Let $(E,(-,-), \circ, \rho)$ be a regular Courant algebroid. And set $F=\rho(E)$.

(1) There exists a splitting $\lambda: F \rightarrow E$ of the short exact sequence

$$
0 \rightarrow \operatorname{ker} \rho \rightarrow E \stackrel{\rho}{\rightarrow} F \rightarrow 0
$$

whose image $\lambda(F)$ is isotropic in $E$.

(2) Given such a splitting $\lambda$, there exists a unique splitting $\sigma_{\lambda}: \mathcal{G} \rightarrow \operatorname{ker} \rho$ of the short exact sequence

$$
0 \rightarrow(\operatorname{ker} \rho)^{\perp} \rightarrow \operatorname{ker} \rho \stackrel{\pi}{\rightarrow} \mathcal{G} \rightarrow 0
$$

with image $\sigma_{\lambda}(\mathcal{G})$ orthogonal to $\lambda(F)$ in $E$.

(3) Given a pair of splittings $\lambda$ and $\sigma_{\lambda}$ as above, the map $\Psi_{\lambda}: F^{*} \oplus \mathcal{G} \oplus$ $F \rightarrow E$ defined by

$$
\Psi_{\lambda}(\xi+\boldsymbol{r}+x)=\frac{1}{2} \Xi^{-1} \rho^{*}(\xi)+\sigma_{\lambda}(\boldsymbol{r})+\lambda(x)
$$

is a dissection of $E$.

Proof. (1) Take any section $\lambda_{0}$ of $\rho: E \rightarrow F$. Consider the bundle map $\varphi: F \rightarrow F^{*}$ defined by

$$
\langle\varphi(x) \mid y\rangle=\left(\lambda_{0}(x), \lambda_{0}(y)\right)
$$

where $x, y \in \Gamma(F)$. Then $\lambda=\lambda_{0}-\frac{1}{2} \Xi^{-1} \rho^{*} \varphi$ is a section of $\rho: E \rightarrow F$ such that

$$
(\lambda x, \lambda y)=0, \quad \forall x, y \in \Gamma(F)
$$


(2) Take any section $\sigma_{0}$ of $\pi: \operatorname{ker} \rho \rightarrow \mathcal{G}$. Consider the bundle map $\psi: \mathcal{G} \rightarrow$ $F^{*}$ defined by

$$
\langle\psi(\boldsymbol{r}) \mid x\rangle=\left(\sigma_{0}(\boldsymbol{r}), \lambda(x)\right),
$$

where $\boldsymbol{r} \in \mathcal{G}$ and $x \in F$. Then $\sigma_{\lambda}=\sigma_{0}-\Xi^{-1} \rho^{*} \psi$ is the only section of $\pi: \operatorname{ker} \rho \rightarrow \mathcal{G}$ such that

$$
\left(\sigma_{\lambda} \boldsymbol{r}, \lambda x\right)=0, \quad \forall \boldsymbol{r} \in \mathcal{G}, x \in \Gamma(F) .
$$

(3) This is obvious.

1.4. Covariant derivatives on regular Courant algebroids. Let us first recall the notion of $E$-connections [1]. Vaisman's metric connections are a related notion $[\mathbf{1 8}]$.

Definition 1.3. An $E$-connection (or covariant $E$-derivative) on $E$ is an $\mathbb{R}$-bilinear map

$$
\nabla^{E}: \Gamma(E) \otimes \Gamma(E) \rightarrow \Gamma(E):(e, s) \mapsto \nabla_{e}^{E} s
$$

such that

$$
\nabla_{f e}^{E} s=f \nabla_{e}^{E} s \quad \text { and } \quad \nabla_{e}^{E}(f s)=f \nabla_{e}^{E} s+(\rho(e) f) s,
$$

for all $f \in C^{\infty}(M)$ and $e, s \in \Gamma(E)$.

The next lemma shows that each regular Courant algebroid $E$ admits E-connections.

If $E$ is a regular Courant algebroid, there always exists a torsion-free connection $\nabla^{F}$ on the integrable distribution $F=\rho(E) \subset T M$. Indeed, it suffices to consider the restriction to $F$ of the Levi-Cività connection of some Riemannian metric on $M$. In the sequel, the symbol $\nabla^{F}$ will be used to denote a chosen torsion-free connection on $F$ and the dual connection on $F^{*}$ as well.

Lemma 1.4. (1) Given a dissection $\Psi$ of a regular Courant algebroid $E$ (identifying $E$ with $F^{*} \oplus \mathcal{G} \oplus F$ ) and the data $\nabla, R$ and $\mathcal{H}$ it induces, each torsion-free connection $\nabla^{F}$ on $F$ determines an E-connection $\nabla^{E}$ on $E$ through the defining relation:

$$
\begin{aligned}
\nabla_{\xi+\boldsymbol{r}+x}^{E}(\eta+\boldsymbol{s}+y)= & \left(\nabla_{x}^{F} \eta-\frac{1}{3} \mathcal{H}(x, y,-)\right) \\
& +\left(\nabla_{x} \boldsymbol{s}+\frac{2}{3}[\boldsymbol{r}, \boldsymbol{s}]^{\mathcal{G}}\right)+\nabla_{x}^{F} y,
\end{aligned}
$$

where $x, y \in \Gamma(F), \xi, \eta \in \Gamma\left(F^{*}\right)$ and $\boldsymbol{r}, \boldsymbol{s} \in \Gamma(\mathcal{G})$.

(2) This covariant derivative $\nabla^{E}$ preserves the pseudo-metric:

$$
\rho\left(e_{1}\right)\left(e_{2}, e_{3}\right)=\left(\nabla_{e_{1}}^{E} e_{2}, e_{3}\right)+\left(e_{2}, \nabla_{e_{1}}^{E} e_{3}\right), \quad \forall e_{1}, e_{2}, e_{3} \in \Gamma(E) .
$$

(3) Moreover, we have

$$
\rho\left(\nabla_{e_{1}}^{E} e_{2}-\nabla_{e_{2}}^{E} e_{1}\right)=\left[\rho\left(e_{1}\right), \rho\left(e_{2}\right)\right] .
$$

We omit the proof as it is straightforward. 
1.5. Naive cohomology. Let $E$ be a Courant algebroid. The nondegenerate pseudo-metric on $E$ induces a nondegenerate pseudo-metric on $\wedge^{k} E$ :

$$
\left(e_{1} \wedge \cdots \wedge e_{k}, f_{1} \wedge \cdots \wedge f_{k}\right)=\left|\begin{array}{cccc}
\left(e_{1}, f_{1}\right) & \left(e_{1}, f_{2}\right) & \cdots & \left(e_{1}, f_{k}\right) \\
\left(e_{2}, f_{1}\right) & \left(e_{2}, f_{2}\right) & \cdots & \left(e_{2}, f_{k}\right) \\
\vdots & \vdots & \ddots & \vdots \\
\left(e_{k}, f_{1}\right) & \left(e_{k}, f_{2}\right) & \cdots & \left(e_{k}, f_{k}\right)
\end{array}\right|
$$

The isomorphism of vector bundles $\wedge^{k} E \rightarrow \wedge^{k} E^{*}$ coming from this nondegenerate pseudo-metric will be denoted by the same symbol $\Xi$. In the sequel, $\wedge^{k} E$ and $\wedge^{k} E^{*}$ are sometimes identified with each other by means of $\Xi$ when it is clear from the context.

The sections of $\wedge^{k} \operatorname{ker} \rho$ are called naive $k$-cochains. The operator

$$
\breve{d}: \Gamma\left(\wedge^{k} \operatorname{ker} \rho\right) \rightarrow \Gamma\left(\wedge^{k+1} \operatorname{ker} \rho\right)
$$

defined by the relation

$$
\begin{aligned}
& \left(\breve{d s}, e_{0} \wedge e_{1} \wedge \cdots \wedge e_{k}\right) \\
& =\sum_{i=0}^{k}(-1)^{i} \rho\left(e_{i}\right)\left(s, e_{0} \wedge \cdots \wedge \widehat{e}_{i} \wedge \cdots \wedge e_{k}\right) \\
& \quad+\sum_{i<j}(-1)^{i+j}\left(s, \llbracket e_{i}, e_{j} \rrbracket \wedge e_{0} \wedge \cdots \wedge \widehat{e_{i}} \wedge \cdots \wedge \widehat{e_{j}} \wedge \cdots, e_{k}\right),
\end{aligned}
$$

where $s \in \Gamma\left(\wedge^{k} \operatorname{ker} \rho\right)$ and $e_{0}, \ldots, e_{k} \in \Gamma(E)$, makes $\left(\Gamma\left(\wedge^{\bullet} \operatorname{ker} \rho\right), \breve{d}\right)$ a cochain complex. Its cohomology $H_{\text {naive }}^{\bullet}(E)$ is called the naive cohomology of the Courant algebroid $E[\mathbf{1 7}]$.

If $E$ is regular, then $\mathcal{A}_{E}=E /(\operatorname{ker} \rho)^{\perp}$ is a regular Lie algebroid. And it is easy to see that the cochain complexes $\left(\Gamma\left(\wedge^{\bullet} \operatorname{ker} \rho\right), \breve{d}\right)$ and $\left(\Gamma\left(\wedge^{\bullet} \mathcal{A}_{E}^{*}\right), d\right)$ are isomorphic. Indeed, we have

$$
\Xi(\operatorname{ker} \rho)=\left((\operatorname{ker} \rho)^{\perp}\right)^{0}=q^{*}\left(\mathcal{A}_{E}^{*}\right)
$$

and

$$
\left(\Xi^{-1} \circ q^{*}\right) \circ d=\breve{d} \circ\left(\Xi^{-1} \circ q^{*}\right) .
$$

Hence, we have the following

Proposition 1.5. For a regular Courant algebroid E, its naive cohomology is isomorphic to the Lie algebroid cohomology of $\mathcal{A}_{E}$.

1.6. A degree-3 characteristic class. The map $K: \Gamma\left(\wedge^{3} E\right) \rightarrow \mathbb{R}$ defined by

$$
K\left(e_{1}, e_{2}, e_{3}\right)=\left(\llbracket e_{1}, e_{2} \rrbracket, e_{3}\right)+\text { c.p. },
$$

where $e_{1}, e_{2}, e_{3} \in \Gamma(E)$, is not a 3 -form on $E$ as it is not $C^{\infty}(M)$-linear. However, it can be modified using an $E$-connection, so that the result is $C^{\infty}(M)$-linear. 
Lemma 1.6 ( [1]). If $\nabla^{E}$ is a covariant derivative on a Courant algebroid E, then

$$
C_{\nabla E}\left(e_{1}, e_{2}, e_{3}\right)=\frac{1}{3}\left(\llbracket e_{1}, e_{2} \rrbracket, e_{3}\right)-\frac{1}{2}\left(\nabla_{e_{1}}^{E} e_{2}-\nabla_{e_{2}}^{E} e_{1}, e_{3}\right)+c . p .,
$$

where $e_{1}, e_{2}, e_{3} \in \Gamma(E)$, defines a 3 -form on $E$.

The following is an analogue of the Chern-Weil construction.

Theorem 1.7. Let $E$ be a regular Courant algebroid.

(1) If $\nabla^{E}$ is the E-connection on $E$ given by equation (1.14), then the 3-form $C_{\nabla E}$ does not depend on the chosen torsion-free connection $\nabla^{F}$, but only on the chosen dissection $\Psi$. It will henceforth be denoted by the symbol $C_{\Psi}$.

(2) The 3-form $C_{\Psi}$ is a naive 3-cocycle.

(3) The cohomology class of $C_{\Psi}$ does not depend on the dissection $\Psi$.

The class of $C_{\Psi}$ is called the characteristic class of the Courant algebroid $E$. The proof of this theorem is deferred to Section 3.

1.7. First Pontryagin class of a quadratic Lie algebroid. We fix a regular Lie algebroid $\left(\mathcal{A},[-,-]_{\mathcal{A}}, a\right)$ over the base manifold $M$, with Lie algebroid cohomology differential operator $d: \Gamma\left(\wedge^{\bullet} \mathcal{A}^{*}\right) \rightarrow \Gamma\left(\wedge^{\bullet+1} \mathcal{A}^{*}\right)$.

We have the short exact sequence of vector bundles

$$
0 \rightarrow \mathcal{G} \rightarrow \mathcal{A} \stackrel{a}{\rightarrow} F \rightarrow 0,
$$

where $F=a(\mathcal{A})$ and $\mathcal{G}=\operatorname{ker} a$.

It is clear that $\mathcal{G}$ is a bundle of Lie algebras, called the Lie-algebra bundle of $\mathcal{A}$. The fiberwise bracket is denoted by $[-,-]^{\mathcal{G}}$.

Definition 1.8. A regular Lie algebroid $\mathcal{A}$ is said to be a quadratic Lie algebroid if the kernel $\mathcal{G}$ of its anchor $a$ is equipped with a fiberwise nondegenerate $a d$-invariant symmetric bilinear form $(-,-)^{\mathcal{G}}$ satisfying:

$$
a(X)(\boldsymbol{r}, \boldsymbol{s})^{\mathcal{G}}=\left([X, \boldsymbol{r}]_{\mathcal{A}}, \boldsymbol{s}\right)^{\mathcal{G}}+\left(\boldsymbol{r},[X, \boldsymbol{s}]_{\mathcal{A}}\right)^{\mathcal{G}}, \quad \forall X \in \Gamma(\mathcal{A}), \boldsymbol{r}, \boldsymbol{s} \in \Gamma(\mathcal{G}) .
$$

Two regular quadratic Lie algebroids $\mathcal{A}_{1}$ and $\mathcal{A}_{2}$ are said to be isomorphic if there is a Lie algebroid isomorphism $\mathcal{A}_{1} \rightarrow \mathcal{A}_{2}$, whose restriction to the kernels of the anchors $\mathcal{G}_{1} \rightarrow \mathcal{G}_{2}$ is an isomorphism of quadratic Lie algebras.

For example, the ample Lie algebroid $\mathcal{A}_{E}=E /(\operatorname{ker} \rho)^{\perp}$ of any regular Courant algebroid $(E,(-,-), \circ, \rho)$ is a quadratic Lie algebroid.

Now a natural question arises as to whether every quadratic Lie algebroid can be realized as the ample Lie algebroid of a Courant algebroid. It turns out that there is an obstruction. To see this, let us introduce some notations.

A hoist of the Lie algebroid $\mathcal{A}$ is a section $\kappa: F \rightarrow \mathcal{A}$ of the anchor $a$. The bundle map $R^{\kappa}: \wedge^{2} F \rightarrow \mathcal{G}$ given by

$$
R^{\kappa}(x, y)=[\kappa(x), \kappa(y)]_{\mathcal{A}}-\kappa([x, y]), \quad \forall x, y \in \Gamma(F)
$$


is its curvature.

Given a quadratic Lie algebroid endowed with a hoist, we can consider the 4 -form $\left\langle R^{\kappa} \wedge R^{\kappa}\right\rangle \in \Gamma\left(\wedge^{4} F^{*}\right)$ given by

$\left\langle R^{\kappa} \wedge R^{\kappa}\right\rangle\left(x_{1}, x_{2}, x_{3}, x_{4}\right)=\frac{1}{4} \sum_{\sigma \in S_{4}} \operatorname{sgn}(\sigma)\left(R^{\kappa}\left(x_{\sigma(1)}, x_{\sigma(2)}\right), R^{\kappa}\left(x_{\sigma(3)}, x_{\sigma(4)}\right)\right)^{\mathcal{G}}$,

for all $x_{1}, x_{2}, x_{3}, x_{4} \in \Gamma(F)$.

Lemma 1.9. The 4-form $\left\langle R^{\kappa} \wedge R^{\kappa}\right\rangle$ is closed and its cohomology class in $H^{4}(F)$ does not depend on the choice of $\kappa$.

Following $[\mathbf{2}, \mathbf{1 5}]$, this cohomology class is called the first Pontryagin class of the quadratic Lie algebroid $\mathcal{A}$.

Theorem 1.10. A regular quadratic Lie algebroid is isomorphic to the ample Lie algebroid of a regular Courant algebroid if and only if its first Pontryagin class vanishes.

The proof of this theorem is deferred to Section 2.2.

\subsection{Characteristic pairs and regular Courant algebroids.}

Definition 1.11. (1) Let $\mathcal{A}$ be a regular quadratic Lie algebroid and let $\kappa$ be a hoist of $\mathcal{A}$. A closed 3 -form $C \in \Gamma\left(\wedge^{3} \mathcal{A}^{*}\right)$ is said to be $\kappa$-coherent if

$$
\begin{gathered}
C(\boldsymbol{r}, \boldsymbol{s}, \boldsymbol{t})=-\left([\boldsymbol{r}, \boldsymbol{s}]^{\mathcal{G}}, \boldsymbol{t}\right)^{\mathcal{G}} \\
C(\boldsymbol{r}, \boldsymbol{s}, \kappa(x))=0 \\
C(\boldsymbol{r}, \kappa(x), \kappa(y))=\left(\boldsymbol{r}, R^{\kappa}(x, y)\right)^{\mathcal{G}},
\end{gathered}
$$

for all $\boldsymbol{r}, \boldsymbol{s}, \boldsymbol{t} \in \mathcal{G}$ and $x, y \in F$. We also say that $(C, \kappa)$ is a coherent pair.

(2) A closed 3-form $C \in \Gamma\left(\wedge^{3} \mathcal{A}^{*}\right)$ on a regular quadratic Lie algebroid $\mathcal{A}$ is called coherent if there exists a hoist $\kappa$ such that $C$ is $\kappa$-coherent.

(3) A characteristic pair is a couple $(\mathcal{A}, C)$ made of a regular quadratic Lie algebroid $\mathcal{A}$ and a coherent 3 -form $C$ on it.

Example 1.12. If $\mathcal{A}=\mathcal{G}$ is a quadratic Lie algebra, then the only coherent 3 -form is the Cartan 3 -form defined by equation (1.18). If $\mathcal{A}=\mathcal{G} \times T M$ is the product Lie algebroid with the obvious quadratic Lie algebroid structure and $\mathcal{H}$ is any closed 3 -form on $M$, then the 3-form $C \in \Gamma\left(\wedge^{3} \mathcal{A}^{*}\right)$ defined by

$$
C(\boldsymbol{r}+x, \boldsymbol{s}+y, \boldsymbol{t}+z)=-\left([\boldsymbol{r}, \boldsymbol{s}]^{\mathcal{G}}, \boldsymbol{t}\right)^{\mathcal{G}}+\mathcal{H}(x, y, z),
$$

for all $\boldsymbol{r}, \boldsymbol{s}, \boldsymbol{t} \in \Gamma(\mathcal{G})$ and $x, y, z \in \Gamma(T M)$, is coherent.

Proposition 1.13. Let $\mathcal{A}$ be a regular quadratic Lie algebroid. The following statements are equivalent.

(1) The first Pontryagin class of $\mathcal{A}$ vanishes. 
(2) There exists a coherent 3 -form on $\mathcal{A}$.

(3) There exists a Courant algebroid whose ample Lie algebroid is $\mathcal{A}$.

Consider the subcomplex $\mathbf{C}_{\leftrightarrow}^{\bullet}(\mathcal{A})$ of $\left(\Gamma\left(\wedge^{\bullet} \mathcal{A}^{*}\right), d\right)$, where $\mathbf{C}_{\leftrightarrow}^{k}(\mathcal{A})$ consists of the sections of $\wedge^{k} \mathcal{A}^{*}$, which are annihilated by all sections of $\wedge^{k} \mathcal{G}$. Its cohomology groups are denoted $H_{\leftrightarrow}^{\bullet}(\mathcal{A})$. Given two coherent 3 -forms $C_{1}$ and $C_{2}$ on $\mathcal{A}$, we have $C_{2}-C_{1} \in \mathbf{C}_{\leftrightarrow}^{3}(\mathcal{A})$.

Definition 1.14. Two characteristic pairs $\left(\mathcal{A}_{1}, C_{1}\right)$ and $\left(\mathcal{A}_{2}, C_{2}\right)$ are said to be equivalent if there exists an isomorphism of quadratic Lie algebroids $\sigma: \mathcal{A}_{1} \rightarrow \mathcal{A}_{2}$ such that $\left[C_{1}-\sigma^{*} C_{2}\right]=0 \in H_{\leftrightarrow}^{3}\left(\mathcal{A}_{1}\right)$. The equivalence class of a characteristic pair $(\mathcal{A}, C)$ will be denoted $[(\mathcal{A}, C)]$.

Our main result is the following:

Theorem 1.15. (1) There is a one-to-one correspondence between regular Courant algebroids up to isomorphism and equivalence classes of characteristic pairs.

(2) If $E$ is a Courant algebroid corresponding to the characteristic pair $(\mathcal{A}, C)$, then the characteristic class of $E$ is equal to $[C] \in H^{3}(\mathcal{A})$.

The proof is postponed to Section 3 .

\section{Standard structures}

2.1. Standard Courant algebroid structures on $\boldsymbol{F}^{*} \oplus \mathcal{G} \oplus \boldsymbol{F}$. Let $F$ be an integrable subbundle of $T M$ and $\mathcal{G}$ be a bundle of quadratic Lie algebras over $M$.

In this section, we are only interested in those Courant algebroid structures on $F^{*} \oplus \mathcal{G} \oplus F$ whose anchor map is

$$
\rho\left(\xi_{1}+\boldsymbol{r}_{1}+x_{1}\right)=x_{1}
$$

whose pseudo-metric is

$$
\left(\xi_{1}+\boldsymbol{r}_{1}+x_{1}, \xi_{2}+\boldsymbol{r}_{2}+x_{2}\right)=\frac{1}{2}\left\langle\xi_{1} \mid x_{2}\right\rangle+\frac{1}{2}\left\langle\xi_{2} \mid x_{1}\right\rangle+\left(\boldsymbol{r}_{1}, \boldsymbol{r}_{2}\right)^{\mathcal{G}},
$$

and whose Dorfman bracket satisfies

$$
\operatorname{Pr}_{\mathcal{G}}\left(\boldsymbol{r}_{1} \circ \boldsymbol{r}_{2}\right)=\left[\boldsymbol{r}_{1}, \boldsymbol{r}_{2}\right]^{\mathcal{G}},
$$

where $\xi_{1}, \xi_{2} \in F^{*}, \boldsymbol{r}_{1}, \boldsymbol{r}_{2} \in \mathcal{G}$, and $x_{1}, x_{2} \in F$. We call them standard Courant algebroid structures on $F^{*} \oplus \mathcal{G} \oplus F$.

Given such a standard Courant algebroid structure on $F^{*} \oplus \mathcal{G} \oplus F$, we define $\nabla, R$, and $\mathcal{H}$ as in equations (1.9)-(1.11). The following Lemma shows that the Dorfman bracket on $\Gamma\left(F^{*} \oplus \mathcal{G} \oplus F\right)$ can be recovered from $\nabla$, $R$, and $\mathcal{H}$. 
Lemma 2.1. Let $\mathcal{P}: \Gamma(\mathcal{G}) \otimes \Gamma(\mathcal{G}) \rightarrow \Gamma\left(F^{*}\right)$ and $\mathcal{Q}: \Gamma(F) \otimes \Gamma(\mathcal{G}) \rightarrow \Gamma\left(F^{*}\right)$ be the maps defined by

$$
\left\langle\mathcal{P}\left(\boldsymbol{r}_{1}, \boldsymbol{r}_{2}\right) \mid y\right\rangle=2\left(\boldsymbol{r}_{2}, \nabla_{y} \boldsymbol{r}_{1}\right)^{\mathcal{G}}
$$

and

$$
\langle\mathcal{Q}(x, \boldsymbol{r}) \mid y\rangle=(\boldsymbol{r}, R(x, y))^{\mathcal{G}} .
$$

Then we have

$$
\begin{aligned}
& x_{1} \circ x_{2}=\mathcal{H}\left(x_{1}, x_{2},-\right)+R\left(x_{1}, x_{2}\right)+\left[x_{1}, x_{2}\right], \\
& \boldsymbol{r}_{1} \circ \boldsymbol{r}_{2}=\mathcal{P}\left(\boldsymbol{r}_{1}, \boldsymbol{r}_{2}\right)+\left[\boldsymbol{r}_{1}, \boldsymbol{r}_{2}\right]^{\mathcal{G}}, \\
& \xi_{1} \circ \boldsymbol{r}_{2}=\boldsymbol{r}_{1} \circ \xi_{2}=\xi_{1} \circ \xi_{2}=0, \\
& x_{1} \circ \xi_{2}=L_{x_{1}} \xi_{2}, \\
& \xi_{1} \circ x_{2}=-L_{x_{2}} \xi_{1}+d^{F}\left\langle\xi_{1} \mid x_{2}\right\rangle, \\
& x_{1} \circ \boldsymbol{r}_{2}=-\boldsymbol{r}_{2} \circ x_{1}=-2 \mathcal{Q}\left(x_{1}, \boldsymbol{r}_{2}\right)+\nabla_{x_{1}} \boldsymbol{r}_{2},
\end{aligned}
$$

for all $\xi_{1}, \xi_{2} \in \Gamma\left(F^{*}\right), \boldsymbol{r}_{1}, \boldsymbol{r}_{2} \in \Gamma(\mathcal{G}), x_{1}, x_{2} \in \Gamma(F)$. Here $d^{F}: C^{\infty}(M) \rightarrow$ $\Gamma\left(F^{*}\right)$ denotes the leafwise de Rham differential.

Proof. Equations (2.6) and (2.8) are quite obvious. To prove equation (2.7), we observe that, for all $x \in \Gamma(F)$,

$$
\begin{aligned}
\left\langle\operatorname{Pr}_{F^{*}}\left(\boldsymbol{r}_{1} \circ \boldsymbol{r}_{2}\right) \mid x\right\rangle & =2\left(\operatorname{Pr}_{F^{*}}\left(\boldsymbol{r}_{1} \circ \boldsymbol{r}_{2}\right), x\right)=2\left(\boldsymbol{r}_{1} \circ \boldsymbol{r}_{2}, x\right)=-2\left(\boldsymbol{r}_{2}, \boldsymbol{r}_{1} \circ x\right) \\
& =2\left(\boldsymbol{r}_{2}, x \circ \boldsymbol{r}_{1}\right)=2\left(\boldsymbol{r}_{2}, \operatorname{Pr}_{\mathcal{G}}\left(x \circ \boldsymbol{r}_{1}\right)\right)=2\left(\boldsymbol{r}_{2}, \nabla_{x} \boldsymbol{r}_{1}\right) .
\end{aligned}
$$

Equation (2.11) can be proved similarly. To prove equation (2.9), we only need to show that $\operatorname{Pr}_{\mathcal{G}}\left(x_{1} \circ \xi_{2}\right)=0$. In fact, for all $\boldsymbol{r} \in \Gamma(\mathcal{G})$,

$$
\begin{aligned}
\left(\operatorname{Pr}_{\mathcal{G}}\left(x_{1} \circ \xi_{2}\right), \boldsymbol{r}\right)^{\mathcal{G}} & =\left(x_{1} \circ \xi_{2}, \boldsymbol{r}\right)=L_{x_{1}}\left(\xi_{2}, \boldsymbol{r}\right)-\left(\xi_{2}, x_{1} \circ \boldsymbol{r}\right) \\
& =-\frac{1}{2}\left\langle\xi_{2} \mid \operatorname{Pr}_{F}\left(x_{1} \circ \boldsymbol{r}\right)\right\rangle=0 .
\end{aligned}
$$

Equation (2.10) follows directly from equation (2.9).

Proposition 2.2. The following identities hold:

$$
\begin{gathered}
L_{x}(\boldsymbol{r}, \boldsymbol{s})^{\mathcal{G}}=\left(\nabla_{x} \boldsymbol{r}, \boldsymbol{s}\right)^{\mathcal{G}}+\left(\boldsymbol{r}, \nabla_{x} \boldsymbol{s}\right)^{\mathcal{G}}, \\
\nabla_{x}[\boldsymbol{r}, \boldsymbol{s}]^{\mathcal{G}}=\left[\nabla_{x} \boldsymbol{r}, \boldsymbol{s}\right]^{\mathcal{G}}+\left[\boldsymbol{r}, \nabla_{x} \boldsymbol{s}\right]^{\mathcal{G}}, \\
\left(\nabla_{x} R(y, z)-R([x, y], z)\right)+c . p .=0, \\
\nabla_{x} \nabla_{y} \boldsymbol{r}-\nabla_{y} \nabla_{x} \boldsymbol{r}-\nabla_{[x, y]} \boldsymbol{r}=[R(x, y), \boldsymbol{r}]^{\mathcal{G}},
\end{gathered}
$$

for all $x, y, z \in \Gamma(F)$ and $\boldsymbol{r}, \boldsymbol{s} \in \Gamma(\mathcal{G})$.

Moreover, we have

$$
d^{F} \mathcal{H}=\langle R \wedge R\rangle
$$


where $\langle R \wedge R\rangle$ denotes the 4-form on $F$ given by

$$
\langle R \wedge R\rangle\left(x_{1}, x_{2}, x_{3}, x_{4}\right)=\frac{1}{4} \sum_{\sigma \in S_{4}} \operatorname{sgn}(\sigma)\left(R\left(x_{\sigma(1)}, x_{\sigma(2)}\right), R\left(x_{\sigma(3)}, x_{\sigma(4)}\right)\right)^{\mathcal{G}}
$$

for any $x_{1}, x_{2}, x_{3}, x_{4} \in F$.

Proof. In light of Lemma 2.1, equation (2.12) follows from

$$
L_{x}(\boldsymbol{r}, \boldsymbol{s})^{\mathcal{G}}=(x \circ \boldsymbol{r}, \boldsymbol{s})^{\mathcal{G}}+(\boldsymbol{r}, x \circ \boldsymbol{s})^{\mathcal{G}},
$$

equation (2.13) from

$$
x \circ(\boldsymbol{r} \circ \boldsymbol{s})=(x \circ \boldsymbol{r}) \circ \boldsymbol{s}+\boldsymbol{r} \circ(x \circ \boldsymbol{s}),
$$

equations (2.14) and (2.16) from

$$
x \circ(y \circ z)=(x \circ y) \circ z+y \circ(x \circ z),
$$

and equation (2.15) from

$$
x \circ(y \circ \boldsymbol{r})=(x \circ y) \circ \boldsymbol{r}+y \circ(x \circ \boldsymbol{r}) .
$$

In summary, we have proved the following:

Theorem 2.3. A Courant algebroid structure on $F^{*} \oplus \mathcal{G} \oplus F$ with the anchor map and pseudo-metric defined by equations (2.1) and (2.2), and satisfying equation (2.3), is completely determined by an $F$-connection $\nabla$ on $\mathcal{G}$, a bundle map $R: \wedge^{2} F \rightarrow \mathcal{G}$, and a 3 -form $\mathcal{H} \in \Gamma\left(\wedge^{3} F^{*}\right)$ satisfying the compatibility conditions (2.12)-(2.16). The Dorfman bracket on $F^{*} \oplus \mathcal{G} \oplus F$ is then given by equations (2.6)-(2.11).

This Courant algebroid structure will be called the standard Courant algebroid determined by the quintuple $(F, \mathcal{G} ; \nabla, R, \mathcal{H})$, and denoted by $E^{s}$.

2.2. Standard quadratic Lie algebroids. We assume again that $F$ is an integrable subbundle of $T M$ and $\mathcal{G}$ is a bundle of quadratic Lie algebras over $M$.

Proposition 2.4. Let $\mathcal{A}$ be a quadratic Lie algebroid with anchor a $: \mathcal{A} \rightarrow$ $T M$ such that $a(\mathcal{A})=F$, and ker $a$ is isomorphic, as a bundle of quadratic Lie algebras, to $\mathcal{G}$. Then any hoist of $\mathcal{A}$ determines an isomorphism $\mathcal{A} \cong$ $\mathcal{G} \oplus F$. The transported Lie bracket on $\Gamma(\mathcal{G} \oplus F)$ is completely determined by an $F$-connection $\nabla$ on $\mathcal{G}$ and a bundle map $R: \wedge^{2} F \rightarrow \mathcal{G}$ satisfying the conditions (2.12) to (2.15), through the following relations:

$$
[\boldsymbol{r}, \boldsymbol{s}]_{\mathcal{A}}=[\boldsymbol{r}, \boldsymbol{s}]^{\mathcal{G}}, \quad[x, y]_{\mathcal{A}}=R(x, y)+[x, y], \quad[x, \boldsymbol{r}]_{\mathcal{A}}=\nabla_{x} \boldsymbol{r},
$$

for all $\boldsymbol{r}, \boldsymbol{s} \in \Gamma(\mathcal{G}), x, y \in \Gamma(F)$. 
This quadratic Lie algebroid $\mathcal{G} \oplus F$ will be called the standard quadratic Lie algebroid determined by the quadruple $(F, \mathcal{G} ; \nabla, R)$, and denoted by $\mathcal{A}^{s}$.

Although it is similar to the proof of Corollary 3.2. in [2], we now sketch a proof of Theorem 1.10 for completeness.

Proof of Theorem 1.10. Given a regular Courant algebroid E, choose a dissection to identify it to $F^{*} \oplus \mathcal{G} \oplus F$. Then, according to equation (2.16), the Pontryagin 4-cocycle of the ample Lie algebroid $\mathcal{A}_{E}$ associated to $E$ is a coboundary. Conversely, given a quadratic Lie algebroid $\mathcal{A}$ with anchor $a: \mathcal{A} \rightarrow T M$ such that $a \mathcal{A}=F$ and ker $a$ is isomorphic to $\mathcal{G}$ as a bundle of quadratic Lie algebras, assume there is a hoist $\kappa$ of the Lie algebroid $\mathcal{A}$ such that the Pontryagin 4-cocycle $\left\langle R^{\kappa} \wedge R^{\kappa}\right\rangle$ is a coboundary, say $d^{F} \mathcal{H}$ for some $\mathcal{H} \in \Gamma\left(\wedge^{3} F^{*}\right)$. We define an $F$-connection $\nabla^{\kappa}$ on $\mathcal{G}$ by setting

$$
\nabla_{x}^{\kappa} \boldsymbol{r}=[\kappa(x), \boldsymbol{r}]_{\mathcal{A}}, \quad \forall x \in \Gamma(F), \boldsymbol{r} \in \Gamma(\mathcal{G}) .
$$

The quintuple $\left(F, \mathcal{G} ; \nabla^{\kappa}, R^{\kappa}, \mathcal{H}\right)$ satisfies all conditions in Theorem 2.3 and it is clear that $\mathcal{A}$ is the ample Lie algebroid associated to the Courant algebroid determined by this quintuple.

2.3. Standard 3-forms on $\mathcal{A}^{s}$ and $\boldsymbol{E}^{\boldsymbol{s}}$. Let $\mathcal{A}^{s}=\mathcal{G} \oplus F$ denote the standard quadratic Lie algebroid determined by the quadruple $(F, \mathcal{G} ; \nabla, R)$. Given any $\mathcal{H} \in \Gamma\left(\wedge^{3} F^{*}\right)$, we define a 3 -form $C^{s} \in \Gamma\left(\wedge^{3}\left(\mathcal{A}^{s}\right)^{*}\right)$ by

$$
\begin{aligned}
C^{s}(\boldsymbol{r}+x, \boldsymbol{s}+y, \boldsymbol{t}+z)= & \mathcal{H}(x, y, z)-\left([\boldsymbol{r}, \boldsymbol{s}]^{\mathcal{G}}, \boldsymbol{t}\right)^{\mathcal{G}}+(R(x, y), \boldsymbol{t})^{\mathcal{G}} \\
& +(R(y, z), \boldsymbol{r})^{\mathcal{G}}+(R(z, x), \boldsymbol{s})^{\mathcal{G}}
\end{aligned}
$$

for all $\boldsymbol{r}, \boldsymbol{s}, \boldsymbol{t} \in \Gamma(\mathcal{G})$ and $x, y, z \in \Gamma(F)$.

Proposition 2.5. The following statements are equivalent:

(1) $\mathcal{H}$ satisfies equation (2.16);

(2) $C^{s}$ is a closed 3 -form;

(3) $C^{s}$ is a coherent 3-form.

Proof. Straightforward calculations lead to

$$
\begin{aligned}
\left(\check{d} C^{s}\right)(x, y, z, w)= & \left(d^{F} \mathcal{H}\right)(x, y, z, w)-\langle R \wedge R\rangle(x, y, z, w), \\
\left(\check{d} C^{s}\right)(x, y, z, \boldsymbol{r})= & \left(\left(\nabla_{x} R(y, z)-R([x, y], z)\right)+\text { c.p., } \boldsymbol{r}\right)^{\mathcal{G}}, \\
\left(\check{d} C^{s}\right)(x, y, \boldsymbol{r}, \boldsymbol{s})= & 0 \\
\left(\check{d} C^{s}\right)(x, \boldsymbol{r}, \boldsymbol{s}, \boldsymbol{t})= & -L_{x}\left([\boldsymbol{r}, \boldsymbol{s}]^{\mathcal{G}}, \boldsymbol{t}\right)^{\mathcal{G}}+\left(\left[\nabla_{x} \boldsymbol{r}, \boldsymbol{s}\right]^{\mathcal{G}}\right. \\
& \left.+\left[\boldsymbol{r}, \nabla_{x} \boldsymbol{s}\right]^{\mathcal{G}}, \boldsymbol{t}\right)^{\mathcal{G}}+\left([\boldsymbol{r}, \boldsymbol{s}]^{\mathcal{G}}, \nabla_{x} \boldsymbol{t}\right)^{\mathcal{G}}, \\
\left(\check{d} C^{s}\right)(\boldsymbol{q}, \boldsymbol{r}, \boldsymbol{s}, \boldsymbol{t})= & 0,
\end{aligned}
$$

for all $x, y, z, w \in \Gamma(F)$ and $\boldsymbol{q}, \boldsymbol{r}, \boldsymbol{s}, \boldsymbol{t} \in \Gamma(\mathcal{G})$.

The right hand side of equations (2.20) and (2.22) vanish due to equations (2.12)-(2.15). Thus $\breve{d} C^{s}=0$ if and only if the right-hand side of equation 
(2.19) vanishes. The latter is equivalent to equation (2.16). This proves the equivalence between the first two statements. The equivalence between the last two statements follows from Definition 1.11 and equation (2.18).

Let $E^{s}=F^{*} \oplus \mathcal{G} \oplus F$ be the standard Courant algebroid determined by the quintuple $(F, \mathcal{G} ; \nabla, R, \mathcal{H})$. Clearly its associated ample quadratic Lie algebroid is the standard quadratic Lie algebroid $\mathcal{A}^{s}$ determined by the quadruple $(F, \mathcal{G} ; \nabla, R)$.

It follows from Proposition 2.5 and the compatibility conditions satisfied by $\nabla, R, \mathcal{H}$ that the form $C^{s}$ defined by equation $(2.18)$ is a closed - and thus coherent -3 -form on $\mathcal{A}^{s}$. Since cocycles of the Lie algebroid $\mathcal{A}^{s}$ can be identified with naive cocycles on $E^{s}$, we obtain a naive 3 -cocycle on $E^{s}$ called the standard 3 -form on $E^{s}$.

At this point, Proposition 1.13 and the following result are quite obvious.

Proposition 2.6. Let $E^{s}$ be the standard Courant algebroid determined by the quintuple $(F, \mathcal{G} ; \nabla, R, \mathcal{H})$, and $C^{s}$ the 3 -form on $\mathcal{A}^{s}$ defined by equation (2.18). Then the characteristic class of $E^{s}$ is equal to $\left[C^{s}\right] \in H^{3}\left(\mathcal{A}^{s}\right)$.

\subsection{Isomorphic standard Courant algebroid structures on $\boldsymbol{F}^{*} \oplus \mathcal{G} \oplus \boldsymbol{F}$.}

Proposition 2.7. Given two different standard Courant algebroid structures $E_{1}^{s}$ and $E_{2}^{s}$ on $F^{*} \oplus \mathcal{G} \oplus F$, any isomorphism of Courant algebroids $\Theta: E_{1}^{s} \rightarrow$ $E_{2}^{s}$ is of the form

$$
\Theta(\xi+\boldsymbol{r}+x)=\left(\xi+\beta(x)-2 \varphi^{*} \tau(\boldsymbol{r})\right)+(\tau(\boldsymbol{r})+\varphi(x))+x,
$$

for $\xi \in F^{*}, \boldsymbol{r} \in \mathcal{G}$ and $x \in F$. Here, $\tau$ is an automorphism of the bundle of quadratic Lie algebras $\mathcal{G}$ and $\varphi: F \rightarrow \mathcal{G}$ and $\beta: F \rightarrow F^{*}$ are bundle maps satisfying the following compatibility conditions:

$$
\begin{aligned}
& \frac{1}{2}\langle\beta(x) \mid y\rangle+\frac{1}{2}\langle x \mid \beta(y)\rangle+(\varphi(x), \varphi(y))^{\mathcal{G}}=0, \\
& \nabla_{x}^{2} \tau(\boldsymbol{r})-\tau \nabla_{x}^{1}(\boldsymbol{r})=[\tau(\boldsymbol{r}), \varphi(x)]^{\mathcal{G}}, \\
& R^{2}(x, y)-\tau R^{1}(x, y)=\tau\left(\nabla_{y}^{1} \tau^{-1} \varphi(x)-\nabla_{x}^{1} \tau^{-1} \varphi(y)\right) \\
& +\varphi[x, y]+[\varphi(x), \varphi(y)]^{\mathcal{G}}, \\
& \mathcal{H}^{2}(x, y, z)-\mathcal{H}^{1}(x, y, z)+2\left(\varphi(x),[\varphi(y), \varphi(z)]^{\mathcal{G}}\right)^{\mathcal{G}} \\
& =\left(2\left(\varphi(x), \tau \nabla_{y}^{1} \tau^{-1} \varphi(z)-\tau R^{1}(y, z)\right)^{\mathcal{G}}\right. \\
& \left.+L_{x}\langle y \mid \beta(z)\rangle+\langle x \mid \beta[y, z]\rangle\right)+ \text { c.p. },
\end{aligned}
$$

for all $x, y, z \in \Gamma(F)$ and $\boldsymbol{r} \in \Gamma(\mathcal{G})$. 
Proof. Consider the bundle maps

$$
\begin{aligned}
\tau: & \mathcal{G} \hookrightarrow E_{1}^{s} \stackrel{\Theta}{\rightarrow} E_{2}^{s} \rightarrow \mathcal{G} ; \\
\varphi: & F \hookrightarrow E_{1}^{s} \stackrel{\Theta}{\rightarrow} E_{2}^{s} \rightarrow \mathcal{G} ; \\
\beta: & F \hookrightarrow E_{1}^{s} \stackrel{\Theta}{\rightarrow} E_{2}^{s} \rightarrow F^{*} ; \\
\gamma: & \mathcal{G} \hookrightarrow E_{1}^{s} \stackrel{\Theta}{\rightarrow} E_{2}^{s} \rightarrow F^{*},
\end{aligned}
$$

where $E_{1}^{s}$ and $E_{2}^{s}$ are both identified with $F^{*} \oplus \mathcal{G} \oplus F$. It is clear that $\Theta(x)=\beta(x)+\varphi(x)+x$, for all $x \in F$. Since $\Theta$ respects the pseudo-metric, we have $(\Theta(x), \Theta(y))=0$, for all $x, y \in F$ and equation (2.25) follows. Since $\Theta(\boldsymbol{r})=\gamma(\boldsymbol{r})+\tau(\boldsymbol{r})$ for all $\boldsymbol{r} \in \mathcal{G}$ and $(\Theta(\boldsymbol{r}), \Theta(x))=(\boldsymbol{r}, x)=0$, we have $\frac{1}{2}\langle\gamma(\boldsymbol{r}) \mid x\rangle+(\tau(\boldsymbol{r}), \varphi(x))^{\mathcal{G}}=0$. Hence $\gamma=-2 \varphi^{*} \tau$. From equation (1.4), it follows that $\Theta(\xi)=\xi$, for all $\xi \in F^{*}$. Thus $\Theta$ must satisfy equation (2.24). Since $\Theta$ takes the Dorfman bracket of $E_{1}^{s}$ to that of $E_{2}^{s}$, equations (2.26) to (2.28) follow from Lemma 2.1.

The following two technical lemmas follow from direct verifications.

Lemma 2.8. Given a bundle map $J: F \rightarrow \mathcal{G}$, define $\Phi_{J} \in \mathbf{C}_{\leftrightarrow}^{2}\left(\mathcal{A}^{s}\right)$ by

$$
\Phi_{J}(\boldsymbol{r}+x, \boldsymbol{s}+y)=(\boldsymbol{r}, J(y))^{\mathcal{G}}-(\boldsymbol{s}, J(x))^{\mathcal{G}},
$$

for all $x, y \in F$ and $\boldsymbol{r}, \boldsymbol{s} \in \mathcal{G}$. Then the differential of $\Phi_{J}$ is given by:

$$
\begin{aligned}
\left(d \Phi_{J}\right) & \left(\boldsymbol{r}_{1}+x_{1}, \boldsymbol{r}_{2}+x_{2}, \boldsymbol{r}_{3}+x_{3}\right) \\
= & \left(\boldsymbol{r}_{1}, \nabla_{x_{3}} J\left(x_{2}\right)-\nabla_{x_{2}} J\left(x_{3}\right)+J\left[x_{2}, x_{3}\right]\right)^{\mathcal{G}} \\
& -\left(J\left(x_{1}\right), R\left(x_{2}, x_{3}\right)+\left[\boldsymbol{r}_{2}, \boldsymbol{r}_{3}\right]^{\mathcal{G}}\right)^{\mathcal{G}}+c . p .,
\end{aligned}
$$

for all $x_{1}, x_{2}, x_{3} \in \Gamma(F)$ and $\boldsymbol{r}_{1}, \boldsymbol{r}_{2}, \boldsymbol{r}_{3} \in \Gamma(\mathcal{G})$.

Lemma 2.9. Given a bundle map $K: F \rightarrow F^{*}$, define $\Psi_{K} \in \mathbf{C}_{\leftrightarrow}^{2}\left(\mathcal{A}^{s}\right)$ by

$$
\Psi_{K}(\boldsymbol{r}+x, \boldsymbol{s}+y)=\langle x \mid K(y)\rangle-\langle y \mid K(x)\rangle,
$$

for all $x, y \in F$ and $\boldsymbol{r}, \boldsymbol{s} \in \mathcal{G}$. Then the differential of $\Psi_{K}$ is given by:

$$
\begin{aligned}
& \left(d \Psi_{K}\right)(\boldsymbol{r}+x, \boldsymbol{s}+y, \boldsymbol{t}+z) \\
& \quad=\left(\left\langle x \mid L_{z} K(y)-L_{y} K(z)+K([y, z])\right\rangle+\langle[x, y] \mid K(z)\rangle\right)+\text { c.p. },
\end{aligned}
$$

for all $x_{1}, x_{2}, x_{3} \in \Gamma(F)$ and $\boldsymbol{r}_{1}, \boldsymbol{r}_{2}, \boldsymbol{r}_{3} \in \Gamma(\mathcal{G})$.

Proposition 2.10. The isomorphism $\Theta: E_{1}^{s} \rightarrow E_{2}^{s}$ of Proposition 2.7 yields an isomorphism $\tilde{\Theta}: \mathcal{A}_{1}^{s} \rightarrow \mathcal{A}_{2}^{s}$ between the associated ample Lie algebroid structures on $\mathcal{G} \oplus F$ :

$$
\tilde{\Theta}(\boldsymbol{r}+x)=(\tau(\boldsymbol{r})+\varphi(x))+x, \quad \forall \boldsymbol{r} \in \mathcal{G}, x \in F .
$$

If $C_{1}^{s}$ and $C_{2}^{s}$ denote the standard coherent 3 -forms (defined in Section 2.3) on $\mathcal{A}_{1}^{s}$ and $\mathcal{A}_{2}^{s}$, respectively, then $\tilde{\Theta}^{*} C_{2}^{s}-C_{1}^{s}=d\left(\frac{1}{2} \Psi_{\beta}+\Phi_{\tau^{-1} \varphi}\right)$. 
Proof. The proof is a direct but tedious calculation using Proposition 2.7, Lemmas 2.8 and 2.9, and equation (2.25).

\section{Proofs of the main theorems}

3.1. Proof of Theorem 1.7. It is easy to see that $C_{\nabla^{E}}$, as defined in equation (1.16), admits the following equivalent formula:

$$
\begin{aligned}
C_{\nabla^{E}}\left(e_{1}, e_{2}, e_{3}\right)= & \left(e_{1} \circ e_{2}, e_{3}\right)-\frac{1}{2} \rho\left(e_{1}\right)\left(e_{2}, e_{3}\right)+\frac{1}{2} \rho\left(e_{2}\right)\left(e_{3}, e_{1}\right) \\
& -\frac{1}{2} \rho\left(e_{3}\right)\left(e_{1}, e_{2}\right)-\frac{1}{2}\left(\nabla_{e_{1}}^{E} e_{2}-\nabla_{e_{2}}^{E} e_{1}, e_{3}\right)+\text { c.p.. }
\end{aligned}
$$

Using the expressions of the Dorfman brackets given by Proposition 2.1, one obtains

$$
\begin{aligned}
C_{\nabla^{E}} & (\xi+\boldsymbol{r}+x, \eta+\boldsymbol{s}+y, \zeta+\boldsymbol{t}+z) \\
= & (R(x, y), \boldsymbol{t})^{\mathcal{G}}+(R(y, z), \boldsymbol{r})^{\mathcal{G}}+(R(z, x), \boldsymbol{s})^{\mathcal{G}} \\
& -\left([\boldsymbol{r}, \boldsymbol{s}]^{\mathcal{G}}, \boldsymbol{t}\right)^{\mathcal{G}}+\mathcal{H}(x, y, z),
\end{aligned}
$$

for all $\xi, \eta, \zeta \in \Gamma\left(F^{*}\right), \boldsymbol{r}, \boldsymbol{s}, \boldsymbol{t} \in \Gamma(\mathcal{G}), x, y, z \in \Gamma(F)$. This shows that $C_{\nabla^{E}}$ coincides with the standard 3-form $C^{s}$ on $E^{s}$ (defined by equation (2.18)). Hence, it is a naive 3-cocycle, and does not depend on the choice of $\nabla^{F}$. Therefore, assertions (1) and (2) are proved.

Finally, we prove assertion (3). Any two dissections $\Psi_{1}$ and $\Psi_{2}$ of $E$ induce two different Courant algebroid structures on $E_{i}^{s} \cong F^{*} \oplus \mathcal{G} \oplus F$, but $E_{1}^{s}$ and $E_{2}^{s}$ are isomorphic. On the other hand, the 3 -form $C_{\Psi_{i}}$ on $E$ is the pull-back of a standard naive 3 -form $\left(C^{s}\right)_{i}$ on $E_{i}^{s}$. Therefore, by Proposition 2.10 and Proposition 1.5, $C_{\Psi_{1}}-C_{\Psi_{2}}$ must be a coboundary $\breve{d} \varphi$, for some naive 2 -form $\varphi$ on $E$.

3.2. Proof of Theorem 1.15. We first show how to construct a Courant algebroid out of a characteristic pair. Let $\mathcal{A}$ be a regular quadratic Lie algebroid and let $(C, \kappa)$ be a coherent pair. Set $F=a(\mathcal{A})$ and $\mathcal{G}=\operatorname{ker} a$. Together, $\mathcal{A}$ and $(C, \kappa)$ induce the $F$-connection $\nabla^{\kappa}$ on $\mathcal{G}$; the bundle map $R^{\kappa}: \wedge^{2} F \rightarrow \mathcal{G}$; and the 3 -form $\mathcal{H}^{(C, \kappa)} \in \Gamma\left(\wedge^{3} F^{*}\right)$ defined by

$$
\mathcal{H}^{(C, \kappa)}(x, y, z)=C(\kappa(x), \kappa(y), \kappa(z)), \quad \forall x, y, z \in \Gamma(F) .
$$

By Proposition 2.5, $C$ being a $\kappa$-coherent 3 -form implies that $\mathcal{H}^{(C, \kappa)}$ satisfies equation (2.16) and thus the quintuple $\left(F, \mathcal{G} ; \nabla^{\kappa}, R^{\kappa}, \mathcal{H}^{(C, \kappa)}\right)$ satisfies the requirements for constructing a standard Courant algebroid structure on the bundle $E^{s}=F^{*} \oplus \mathcal{G} \oplus F$ described by Theorem 2.3. We shall denote such a Courant algebroid by $E^{s}(\mathcal{A} ; C, \kappa)$, or simply just $E^{s}(C, \kappa)$ if $\mathcal{A}$ is obvious from the context.

In the sequel, the isomorphism class of a regular Courant algebroid $E$ will be denoted by $[E]$. Similarly, the equivalence class of a characteristic pair $(\mathcal{A}, C)$ is denoted by $[(\mathcal{A}, C)]$. 
Define a map $i$ from the set of equivalence classes of characteristic pairs to the set of isomorphism classes of regular Courant algebroids sending $[(\mathcal{A}, C)]$ to $\left[E^{s}(\mathcal{A} ; C, \kappa)\right]$. Here $\kappa$ is any hoist of $\mathcal{A}$ such that $(C, \kappa)$ is a coherent pair on $\mathcal{A}$.

Proposition 3.1. The map $\mathfrak{i}([(\mathcal{A}, C)])=\left[E^{s}(\mathcal{A} ; C, \kappa)\right]$ is well-defined.

Before we prove this proposition, let us establish some useful facts. First, we study the change of hoists. It is obvious that for any two hoists of $\mathcal{A}$ : $\kappa$ and $\kappa^{\prime}$, there exists a unique map $J: F \rightarrow \mathcal{G}$ such that $\kappa^{\prime}(x)=\kappa(x)-J(x)$ for all $x \in F$. Recall the notation $\nabla^{\kappa}$ defined by equation (2.17). It is easy to check that

$$
\begin{gathered}
\nabla_{x}^{\kappa^{\prime}} \boldsymbol{r}-\nabla_{x}^{\kappa} \boldsymbol{r}=[\boldsymbol{r}, J(x)]^{\mathcal{G}}, \\
R^{\kappa^{\prime}}(x, y)-R^{\kappa}(x, y)=[J(x), J(y)]-\nabla_{x}^{\kappa} J(y)+\nabla_{y}^{\kappa} J(x)+J[x, y],
\end{gathered}
$$

for all $x, y \in \Gamma(F), \boldsymbol{r} \in \Gamma(\mathcal{G})$.

By Lemma 2.8 and the above two equalities, one can easily prove the following lemma.

Lemma 3.2. If $(C, \kappa)$ is a coherent pair, then so is the pair $\left(C+d \Phi_{J}, \kappa-J\right)$. Here $\Phi_{J}$ is a 2 -form on $\mathcal{A}$ defined by equation (2.29).

An important fact is the following:

Lemma 3.3. The Courant algebroids $E^{s}(C, \kappa)$ and $E^{s}\left(C+d \Phi_{J}, \kappa-J\right)$ are isomorphic.

Proof. Write $C^{1}=C, C^{2}=C+d \Phi_{J}, \kappa^{1}=\kappa, \kappa^{2}=\kappa-J$ and let $E_{i}=E^{s}\left(C^{i}, \kappa^{i}\right)$. Clearly $E_{i}$ is determined by the quintuple $\left(F, \mathcal{G} ; \nabla^{i}, R^{i}, \mathcal{H}^{i}\right)$, where $\nabla^{i}=\nabla^{\kappa^{i}}, R^{i}=R^{\kappa^{i}}$ and $\mathcal{H}^{i}=\mathcal{H}^{\left(C^{i}, \kappa^{i}\right)}$. We have the following equalities:

$$
\begin{aligned}
\nabla_{x}^{2} \boldsymbol{r}-\nabla_{x}^{1} \boldsymbol{r}= & {[\boldsymbol{r}, J(x)]^{\mathcal{G}}, } \\
R^{2}(x, y)-R^{1}(x, y)= & {[J(x), J(y)]^{\mathcal{G}}-\nabla_{x}^{1} J(y)+\nabla_{y}^{1} J(x)+J[x, y], } \\
\mathcal{H}^{2}(x, y, z)-\mathcal{H}^{1}(x, y, z)= & \left(J(x), \nabla_{y}^{1} J(z)-\nabla_{z}^{1} J(y)-J[y, z]-2 R^{1}(y, z)\right)^{\mathcal{G}} \\
& -2\left([J(x), J(y)]^{\mathcal{G}}, J(z)\right)^{\mathcal{G}}+\text { c.p. },
\end{aligned}
$$

for all $x, y, z \in \Gamma(F), r \in \Gamma(\mathcal{G})$. Define a map $I: E_{1} \rightarrow E_{2}$ by

$$
I(\xi+\boldsymbol{r}+x)=\left(\xi+\beta(x)-2 J^{*}(\boldsymbol{r})\right)+(\boldsymbol{r}+J(x))+x,
$$

where $\xi \in F^{*}, r \in \mathcal{G}, x \in F$, and $\beta$ denotes the map $-J^{*} \circ J$. According to Proposition 2.7 and the above equalities, $I$ is a Courant algebroid isomorphism.

Lemma 3.4. Assume that $(C, \kappa)$ and $\left(C+d \Phi_{J}, \kappa\right)$ are both coherent pairs, for some bundle map $J: F \rightarrow \mathcal{G}$. Then the Courant algebroids $E^{s}(C, \kappa)$ and $E^{s}\left(C+d \Phi_{J}, \kappa\right)$ are isomorphic. 
Proof. Let $E_{1}=E^{s}(C, \kappa), E_{2}=E^{s}\left(C+d \Phi_{J}, \kappa\right)$. Clearly, $E_{i}$ is the standard Courant algebroid determined by the data $\nabla=\nabla^{\kappa}, R=R^{\kappa}$ and $\mathcal{H}^{i}$, where $\mathcal{H}^{1}=\mathcal{H}^{(C, \kappa)}, \mathcal{H}^{2}=\mathcal{H}^{\left(C+d \Phi_{J}, \kappa\right)}$. Since $(C, \kappa)$ and $\left(C+d \Phi_{J}, \kappa\right)$ are both coherent pairs, we have $\iota_{\boldsymbol{r}}\left(d \Phi_{J}\right)=0$, for all $\boldsymbol{r} \in \mathcal{G}$. By Lemma 2.8, one can check that this is equivalent to the following two conditions:

$$
J(F) \subset Z(\mathcal{G}) \quad \text { and } \quad \nabla_{x} J(y)-\nabla_{y} J(x)-J[x, y]=0, \quad \forall x, y \in \Gamma(F) .
$$

Using these, one has

$$
\mathcal{H}^{2}(x, y, z)-\mathcal{H}^{1}(x, y, z)=-(J(x), R(y, z))^{\mathcal{G}}+\text { c.p. },
$$

for all $x, y, z \in \Gamma(F)$. Then by Proposition 2.7, the map $I: E_{1} \rightarrow E_{2}$ defined by

$$
I(\xi+\boldsymbol{r}+x)=\left(\xi+\beta(x)-2 \varphi^{*}(\boldsymbol{r})\right)+(\boldsymbol{r}+\varphi(x))+x,
$$

where $\xi \in F^{*} ; \boldsymbol{r} \in \mathcal{G} ; x \in F ; \varphi=\frac{1}{2} J$; and $\beta=-\frac{1}{4} J^{*} \circ J$, is an isomorphism of Courant algebroids.

The following Corollary implies that $\left[E^{s}(\mathcal{A} ; C, \kappa)\right]$ actually does not depend on the choice of the hoist $\kappa$ of $\mathcal{A}$.

Corollary 3.5. If $(C, \kappa)$ and $\left(C, \kappa^{\prime}\right)$ are both coherent pairs on $\mathcal{A}$, then the Courant algebroids $E^{s}(C, \kappa)$ and $E^{s}\left(C, \kappa^{\prime}\right)$ are isomorphic.

Proof. Suppose that $\kappa^{\prime}=\kappa+J$, for some bundle map $J: F \rightarrow \mathcal{G}$. Then according to Lemma 3.2 , the pair $\left(C+d \Phi_{J}, \kappa\right)$ is also coherent. By Lemma 3.3 and 3.4, we have isomorphisms $E^{s}\left(C, \kappa^{\prime}\right) \cong E^{s}\left(C+d \Phi_{J}, \kappa\right) \cong E^{s}(C, \kappa)$.

Lemma 3.6. If $(C, \kappa)$ is a coherent pair, so is the pair $\left(C+a^{*}\left(d^{F} \omega\right), \kappa\right)$, for any 2 -form $\omega \in \Gamma\left(\wedge^{2} F^{*}\right)$. Moreover, the Courant algebroids $E^{s}(C, \kappa)$ and $E^{s}\left(C+a^{*}\left(d^{F} \omega\right), \kappa\right)$ are isomorphic.

Proof. It is clear that $\iota_{\boldsymbol{r}}\left(a^{*}\left(d^{F} \omega\right)\right)=0, \forall \boldsymbol{r} \in \mathcal{G}$. Thus one can easily check that $\left(C+a^{*}\left(d^{F} \omega\right), \kappa\right)$ is a coherent pair. Let $E_{i}$ be the standard Courant algebroid determined by the quintuple $\left(F, \mathcal{G} ; \nabla, R, \mathcal{H}^{i}\right)$, where $\mathcal{H}^{1}=\mathcal{H}^{(C, \kappa)}$, $\mathcal{H}^{2}=\mathcal{H}^{\left(C+a^{*}\left(d^{F} \omega\right), \kappa\right)}$. Obviously, $\mathcal{H}^{2}-\mathcal{H}^{1}=d^{F} \omega$. By Proposition 2.7, we can construct an isomorphism $I: E_{1} \rightarrow E_{2}$ by setting

$$
I(\xi+\boldsymbol{r}+x)=\left(\xi-\omega^{\sharp}(x)\right)+\boldsymbol{r}+x,
$$

for $\xi \in F^{*}, \boldsymbol{r} \in \mathcal{G}$, and $x \in F$. Here $\omega^{\sharp}: F \rightarrow F^{*}$ is defined by

$$
\left\langle\omega^{\sharp}(x) \mid y\right\rangle=\omega(x, y), \quad \forall x, y \in \Gamma(F) .
$$

Lemma 3.7. Let $\left(C_{1}, \kappa_{1}\right)$ and $\left(C_{2}, \kappa_{2}\right)$ be two coherent pairs. Assume that $C_{2}=C_{1}+d \varphi$, for some $\varphi \in \mathbf{C}_{\leftrightarrow}^{2}(\mathcal{A})$. Then the Courant algebroids $E^{s}\left(C_{1}, \kappa_{1}\right)$ and $E^{s}\left(C_{2}, \kappa_{2}\right)$ are isomorphic. 
Proof. Choose $J: F \rightarrow \mathcal{G}$ so that $\kappa_{2}=\kappa_{1}+J$. Lemma 3.3 implies that

$$
\begin{aligned}
E^{s}\left(C_{2}, \kappa_{2}\right) & =E^{s}\left(C_{2}, \kappa_{1}+J\right) \cong E^{s}\left(C_{2}+d \Phi_{J}, \kappa_{1}\right) \\
& =E^{s}\left(C_{1}+d\left(\varphi+\Phi_{J}\right), \kappa_{1}\right) .
\end{aligned}
$$

The hoist $\kappa_{1}$ produces a decomposition $\mathcal{A} \cong \mathcal{G} \oplus F$. Hence, there exist $J^{\prime}: F \rightarrow \mathcal{G}$ and $\omega \in \Gamma\left(\wedge^{2} F^{*}\right)$ such that $\varphi+\Phi_{J}=\Phi_{J^{\prime}}+a^{*} \omega$. By Lemmas 3.6 and 3.4 , we have

$$
\begin{aligned}
E^{s}\left(C_{1}+d\left(\varphi+\Phi_{J}\right), \kappa_{1}\right) & =E^{s}\left(C_{1}+d \Phi_{J^{\prime}}+a^{*}\left(d^{F} \omega\right), \kappa_{1}\right) \\
& \cong E^{s}\left(C_{1}+d \Phi_{J^{\prime}}, \kappa_{1}\right) \cong E^{s}\left(C_{1}, \kappa_{1}\right) .
\end{aligned}
$$

Proof of Proposition 3.1. First we show that $\mathfrak{i}$ is well defined. Assume that two characteristic pairs $(\mathcal{A}, C)$ and $(\overline{\mathcal{A}}, \bar{C})$ are equivalent, i.e., there is an isomorphism $\sigma: \mathcal{A} \rightarrow \overline{\mathcal{A}}$ such that $C-\sigma^{*} \bar{C}=d \varphi$, for some $\varphi \in \mathbf{C}_{\leftrightarrow}^{2}(\mathcal{A})$. We need to show that there is an isomorphism of Courant algebroids from $E^{s}(\mathcal{A} ; C, \kappa)$ to $E^{s}(\overline{\mathcal{A}} ; \bar{C}, \bar{\kappa})$. It is quite obvious that $\left(\sigma^{*} \bar{C}, \sigma^{-1} \circ \bar{\kappa}\right)$ is also a coherent pair on $\mathcal{A}$ and that $E^{s}\left(\mathcal{A} ; \sigma^{*} \bar{C}, \sigma^{-1} \circ \bar{\kappa}\right)$ is isomorphic to $E^{s}(\overline{\mathcal{A}} ; \bar{C}, \bar{\kappa})$. Since $C-\sigma^{*} \bar{C}=d \varphi$, Lemma 3.7 gives an isomorphism between $E^{s}(\mathcal{A} ; C, \kappa)$ and $E^{s}\left(\mathcal{A} ; \sigma^{*} \bar{C}, \sigma^{-1} \circ \bar{\kappa}\right)$. Thus we conclude that $E^{s}(\mathcal{A} ; C, \kappa)$ is isomorphic to $E^{s}(\overline{\mathcal{A}} ; \bar{C}, \bar{\kappa})$ and $\mathfrak{i}$ is well defined.

With these preparations, we are able to prove the second main theorem in this paper.

Proof of Theorem 1.15. (a) Let us denote by $\mathfrak{R}$ the set of isomorphism classes of regular Courant algebroids, and by $\mathfrak{C}$ the set of equivalence classes of characteristic pairs. We need to establish a one-to-one correspondence between $\mathfrak{R}$ and $\mathfrak{C}$.

For any regular Courant algebroid $E$, one may choose a dissection so as to identify $E$ with $E^{s}=F^{*} \oplus \mathcal{G} \oplus F$. In Section 2.2, we showed that there is a standard naive 3 -cocycle $C^{s}$ on $E^{s}$ and it corresponds to a coherent 3 -form on $\mathcal{A}^{s}=\mathcal{G} \oplus F$, also denoted by $C^{s}$.

Hence we can define a map $\mathfrak{b}: \mathfrak{R} \rightarrow \mathfrak{C},[E] \mapsto\left[\left(\mathcal{A}^{s}, C^{s}\right)\right]$. By Proposition 2.10, this map is well defined and does not depend on the choice of the dissection.

On the other hand, Proposition 3.1 indicates that there is a well-defined map $\mathfrak{i}: \mathfrak{C} \rightarrow \mathfrak{R}$. An easy verification shows that $\mathfrak{i}$ is indeed the inverse of $\mathfrak{b}$. This completes the proof.

(b) This follows from Proposition 2.6.

\subsection{Classification.}

Lemma 3.8. Let $C \in \Gamma\left(\wedge^{3} \mathcal{A}^{*}\right)$ be a coherent 3-form. Then any other coherent 3 -form is of the form $C+a^{*}(\varpi)+d \Phi_{J}$, where $\varpi \in \Gamma\left(\wedge^{3} F^{*}\right)$ is a closed 3 -form on $F$ and $\Phi_{J}$ is defined by equation (2.29). 
Proof. Assume that $C$ (resp. $C^{\prime}$ ) is $\kappa$ (resp. $\kappa^{\prime}$ )-coherent, for some hoist $\kappa$ (resp. $\kappa^{\prime}$ ) of $\mathcal{A}$. The difference between $\kappa$ and $\kappa^{\prime}$ determines a bundle map $J: F \rightarrow \mathcal{G}$ such that $\kappa^{\prime}=\kappa-J$. A direct calculation shows that the 3 -form $C^{\prime}-C-d \Phi_{J}$ must be the pull back of a certain 3 -form $\varpi$ by the anchor map $a$. Conversely, it is easy to verify that $C+a^{*}(\varpi)+d \Phi_{J}$ is coherent with respect to $\kappa^{\prime}=\kappa-J$.

Definition 3.9. A 3 -form $\vartheta \in \mathbf{C}_{\leftrightarrow}^{3}(\mathcal{A})$ of a quadratic Lie algebroid $\mathcal{A}$ is said to be intrinsic, if there exists a coherent 3 -form $C$ and an automorphism $\sigma: \mathcal{A} \rightarrow \mathcal{A}$ over the identity such that $\vartheta=\sigma^{*} C-C$.

It is simple to see that every intrinsic 3 -form is closed, and therefore defines a class in $H_{\leftrightarrow}^{3}(\mathcal{A})$. It turns out that this is the obstruction class to lifting the automorphism $\sigma$ to a Courant algebroid automorphism.

Lemma 3.10. Let $\mathcal{A}_{E}$ be the ample Lie algebroid of a regular Courant algebroid $E$ and $\vartheta=\sigma^{*} C-C$ an intrinsic 3 -form of $\mathcal{A}_{E}$. Then $[\vartheta]=0$ if and only if $\sigma$ can be lifted to an automorphism $\tilde{\sigma}$ of the Courant algebroid $E$.

Let $\mathbb{I}$ denote the subset in $H_{\leftrightarrow}^{3}(\mathcal{A})$ consisting of the cohomology classes of all intrinsic 3 -forms. The following result is an easy consequence of Lemma 3.8.

Proposition 3.11. The set $\mathbb{I}$ is an abelian subgroup of $a^{*} H^{3}(F)$ consisting of the cohomology classes $\left[\sigma^{*} C_{0}-C_{0}\right]$ for a fixed coherent 3 -form $C_{0}$ and all automorphisms $\sigma: \mathcal{A} \rightarrow \mathcal{A}$ over the identity.

Hence the abelian group $\mathbb{I}$ captures the essentially nontrivial automorphisms of $\mathcal{A}$, i.e., those automorphisms that cannot be lifted to automorphisms on the Courant algebroid level.

Let $\Phi$ be the natural map from the isomorphism classes of regular Courant algebroids to the isomorphism classes of quadratic Lie algebroids with vanishing first Pontryagin class. We are now ready to prove the last main theorem of this paper.

Theorem 3.12. For any quadratic Lie algebroid $\mathcal{A}$ with vanishing first Pontryagin class, $\Phi^{-1}([\mathcal{A}])$ is isomorphic to

$$
\frac{a^{*} H^{3}(F)}{\mathbb{I}} \cong \frac{H^{3}(F)}{\left(a^{*}\right)^{-1}(\mathbb{I})} .
$$

Proof. By Theorem 1.15, we understand that every isomorphism class of Courant algebroid in $\Phi^{-1}([\mathcal{A}])$ can be characterized by an equivalence class of characteristic pairs $(\mathcal{A}, C)$, where $C$ is a coherent 3 -form. Lemma 3.8 implies that $a^{*}\left(H^{3}(F)\right)$ acts transitively on $\Phi^{-1}([\mathcal{A}])$. Now we determine the isotropy group of this action. Assume that $\varpi \in \Gamma\left(\wedge^{3} F^{*}\right)$ is a closed 3 -form such that the characteristic pairs $(\mathcal{A}, C)$ and $\left(\mathcal{A}, C+a^{*}(\varpi)\right)$ are 
equivalent. By definition, there exists an automorphism $\sigma$ of $\mathcal{A}$ such that $\left[\sigma^{*}(C)-\left(C+a^{*}(\varpi)\right)\right]=0$ in $H_{\leftrightarrow}^{3}(\mathcal{A})$. Hence, it follows that $\left[a^{*}(\varpi)\right] \in \mathbb{I}$. Conversely, it is simple to see that $\mathbb{I}$ acts trivially on $\Phi^{-1}([\mathcal{A}])$. This concludes the proof of the theorem.

The group $\left(a^{*}\right)^{-1}(\mathbb{I})$ is called the intrinsic group of $\mathcal{A}$. Note that the classification problem investigated here differs from that studied by Ševera and Bressler even in the transitive case. Ševera and Bressler classified the isomorphism classes of transitive Courant algebroid extensions of a given Lie algebroid $[\mathbf{2}, \mathbf{1 5}]$, while we are interested in isomorphism classes of Courant algebroids themselves.

Remark 3.13. Note that $a^{*}: H^{3}(F) \rightarrow H_{\leftrightarrow}^{3}(\mathcal{A})$ is not necessarily injective.

Let $M$ be a contact manifold with $\theta \in \overleftrightarrow{\Omega^{1}}(M)$ being the contact 1 -form. Consider the central extension $\mathcal{A}=(\mathbb{R} \times M) \oplus T M$, of the Lie algebroid $T M$ by the exact 2 -form $d \theta$ considered as a 2 -cocycle of the Lie algebroid $T M$. It is a transitive Lie algebroid. Endow $\mathbb{R} \times M \rightarrow M$ with its standard fiberwise metric. Clearly $\mathcal{A}$ is a quadratic Lie algebroid. It is easy to check that $a^{*}[\theta \wedge d \theta]=0 \in H_{\leftrightarrow}^{3}(\mathcal{A})$. However $[\theta \wedge d \theta]$ is a nontrivial class in $H_{\mathrm{DR}}^{3}(M)$.

For instance, if $M=S^{3}$ with the canonical contact structure, $H_{\mathrm{DR}}^{3}(M) \cong$ $\mathbb{R}$ and $a^{*} H_{\mathrm{DR}}^{3}(M)=0$. As a consequence, there is a unique Courant algebroid (up to isomorphism) whose induced quadratic Lie algebroid is isomorphic to $\mathcal{A}=(\mathbb{R} \times M) \oplus T M$. On the other hand, according to $[\mathbf{2}, \mathbf{1 5}]$, Courant algebroid extensions of $\mathcal{A}=(\mathbb{R} \times M) \oplus T M$ are parameterized by $\mathbb{R}$.

In general, it is hard to describe explicitly the intrinsic group $\left(a^{*}\right)^{-1}(\mathbb{I})$. The following example, due to Ševera [15], gives a nice description of the intrinsic group in a special case. We are grateful to Ševera, who pointed out this example to us, which led us to find an error in an early version of the draft.

Example 3.14. Let $\mathfrak{g}$ be a compact Lie algebra such that both $\operatorname{Out}(\mathfrak{g})$ and $Z(\mathfrak{g})$ are trivial. Assume that $M$ is a connected and simply connected manifold. Choose an ad-invariant nondegenerate bilinear form on $\mathfrak{g}$. Consider the canonical quadratic Lie algebroid $\mathcal{A}=(M \times \mathfrak{g}) \oplus T M$. The Cartan 3-form induces a coherent 3 -form $C \in \Gamma\left(\wedge^{3} \mathcal{A}^{*}\right)$ :

$$
C(\boldsymbol{r}+x, \boldsymbol{s}+y, \boldsymbol{t}+z)=-\left([\boldsymbol{r}, \boldsymbol{s}]^{\mathfrak{g}}, \boldsymbol{t}\right),
$$

$\forall \boldsymbol{r}, \boldsymbol{s}, \boldsymbol{t} \in C^{\infty}(M, \mathfrak{g})$ and $x, y, z \in \Gamma(T M)$.

Let $G$ be a connected and simply connected Lie group whose Lie algebra is $\mathfrak{g}$. Then any automorphism $\sigma$ of $\mathcal{A}$ is determined by a smooth map $\tau: M \rightarrow$ $G$, where $\sigma$ can be written as

$$
\sigma(\boldsymbol{r}+v)=\operatorname{Ad}_{\tau(x)}(\boldsymbol{r}-J(v))+v, \forall \boldsymbol{r} \in \mathfrak{g}, v \in T_{x} M .
$$


Here $J: T M \rightarrow \mathfrak{g}$ is the bundle map defined by:

$$
J(v)=L_{\tau(x)^{-1}}\left(\tau_{*}(v)\right), \quad \forall v \in T_{x} M .
$$

To see this, one can consider $\mathcal{A}$ as the Atiyah Lie algebroid of the trivial principal bundle $M \times G \rightarrow M$. Then $\sigma$ is induced by the principal bundle automorphism defined by the gauge group element $\tau: M \rightarrow G$.

Using Lemma 2.8, one obtains

$\left(\sigma^{*} C-C\right)(\boldsymbol{r}+x, \boldsymbol{s}+y, \boldsymbol{t}+z)=-\left(d \Phi_{J}\right)(\boldsymbol{r}+x, \boldsymbol{s}+y, \boldsymbol{t}+z)+\left([J(x), J(y)]^{\mathfrak{g}}, J(z)\right)$.

It thus follows that

$$
\left[\sigma^{*} C-C\right]=-(J \circ a)^{*}[\alpha]=-a^{*}\left(J^{*}[\alpha]\right),
$$

where $\alpha=([\cdot, \cdot], \cdot) \in \wedge^{3} \mathfrak{g}^{*}$. It is simple to see that $J^{*}(\alpha)=\tau^{*}(\tilde{\alpha})$, where $\tilde{\alpha}$ is the corresponding Cartan 3 -form on $G$. As a consequence, the intrinsic group $\left(a^{*}\right)^{-1}(\mathbb{I})$ is generated by elements of the form $\tau^{*}[\tilde{\alpha}]$, where $\tau: M \rightarrow G$ is any smooth map. If $\tau_{1}$ and $\tau_{2}$ are homotopic, $\tau_{1}^{*}[\tilde{\alpha}]=\tau_{2}^{*}[\tilde{\alpha}]$. Hence $\left(a^{*}\right)^{-1}(\mathbb{I})$ must be discrete.

Here is one example. Let $M=G=S U(2)$. Then $H_{\mathrm{DR}}^{3}(M) \cong \mathbb{R}$ and the intrinsic group $\left(a^{*}\right)^{-1}(\mathbb{I})$ is isomorphic to $\mathbb{Z}$ with the generator being $[\tilde{\alpha}]$. Hence by Theorem 3.12, the isomorphism classes of Courant algebroids with ample Lie algebroid isomorphic to the quadratic Lie algebroid $\left(S^{3} \times\right.$ $\mathfrak{s u}(2)) \oplus T S^{3}$ is parameterized by $\mathbb{R} / \mathbb{Z} \cong S^{1}$.

\section{References}

[1] A. Alekseev and P. Xu, Derived brackets and courant algebroids, http://www.math . psu.edu/ping/anton-final.pdf (2001).

[2] P. Bressler, The first Pontryagin class, Compos. Math. 143(5) (2007), 1127-1163. MR 2360313 (2009f:57041)

[3] T.J. Courant, Dirac manifolds, Trans. Amer. Math. Soc. 319(2) (1990), 631-661. MR 998124 (90m:58065)

[4] M. Crainic and R.L. Fernandes, Secondary characteristic classes of Lie algebroids, Quantum field theory and noncommutative geometry, Lecture Notes in Phys., 662, Springer, Berlin, 2005, pp. 157-176. MR 2179182 (2007b:53047)

[5] S. Evens, J.-H. Lu, and A. Weinstein, Transverse measures, the modular class and a cohomology pairing for Lie algebroids, Quart. J. Math. Oxford Ser. (2) 50(200) (1999), 417-436. MR 1726784 (2000i:53114)

[6] R.L. Fernandes, Lie algebroids, holonomy and characteristic classes, Adv. Math. 170(1) (2002), 119-179. MR 1929305 (2004b:58023)

[7] G. Ginot and M. Grützmann, Cohomology of Courant algebroids with split base, J. Symplectic Geom. 7(3) (2009), 311-335. MR 2534188 (2010g:53157)

[8] A. Gracia-Saz and R.A. Mehta, Lie algebroid structures on double vector bundles and representation theory of Lie algebroids, Adv. Math. 223(4) (2010), 1236-1275. MR 2581370 (2011j:53162)

[9] M. Grützmann and M. Stiénon, Matched pairs of courant algebroids, arXiv:math/ 1204.1311 (2012). 
[10] Z.-J. Liu, A. Weinstein, and P. Xu, Manin triples for Lie bialgebroids, J. Differential Geom. 45(3) (1997), 547-574. MR 1472888 (98f:58203)

[11] K.C.H. Mackenzie, General theory of Lie groupoids and Lie algebroids, London Mathematical Society Lecture Note Series, 213, Cambridge University Press, Cambridge, 2005. MR 2157566 (2006k:58035)

[12] D. Roytenberg, Courant algebroids, derived brackets and even symplectic supermanifolds, arXiv:math/9910078 (1999).

[13] _ On the structure of graded symplectic supermanifolds and Courant algebroids, Quantization, Poisson brackets and beyond (Manchester, 2001), Contemp. Math., vol. 315, Amer. Math. Soc., Providence, RI, 2002, pp. 169-185. MR 1958835 (2004i:53116)

[14] D. Roytenberg and A. Weinstein, Courant algebroids and strongly homotopy Lie algebras, Lett. Math. Phys. 46(1) (1998), 81-93. MR 1656228 (2000c:17036)

[15] P. Ševera, First and fourth letters to Alan Weinstein, http://sophia.dtp.fmph. uniba.sk/ severa/letters.

[16] J. Stasheff, Differential graded Lie algebras, quasi-Hopf algebras and higher homotopy algebras, Quantum groups (Leningrad, 1990), Lecture Notes in Math., 1510, Springer, Berlin, 1992, pp. 120-137. MR 1183483 (93j:17055)

[17] M. Stiénon and P. Xu, Modular classes of Loday algebroids, C. R. Math. Acad. Sci. Paris 346(3-4) (2008), 193-198. MR 2393640 (2009g:53125)

[18] I. Vaisman, Transitive Courant algebroids, Int. J. Math. Math. Sci. (11) (2005), 17371758. MR 2178250 (2006f:53124)

Department of Mathematics, Tsinghua University, Beijing, People's Republic of China

E-mail address: zchen@math.tsinghua.edu.cn

Department of Mathematics, Penn State University, University Park,

PA 16802, USA

E-mail address: stienon@math.psu.edu

E-mail address: ping@math.psu.edu

Received: 12/04/2009, accepted 03/12/2012

The authors thank Camille Laurent-Gengoux, Zhang-Ju Liu, Jim Stasheff and Alan Weinstein for useful discussions and comments. The authors are indebted to Pavol Ševera, who provided Example 3.14, and called their attention to [15], which led to the correction of an error in an early version of the paper. Zhuo Chen is also grateful to the MaxPlanck-Institut für Mathematik for providing him with excellent working conditions and a friendly environment. Research partially supported by NSF grants DMS-0605725 and DMS-0801129, and NSFC grant 11001146 and SQKM201211232017. 